\title{
Operator Expansions and Constrained Quadratic Optimization for Interface Reconstruction: Impenetrable Periodic Acoustic Media
}

\author{
Alison Malcolm \\ Department of Earth, Atmospheric, \\ and Planetary Sciences \\ Massachusetts Institute of Technology \\ Cambridge, MA 02139 \\ David P. Nicholls \\ Department of Mathematics, Statistics, \\ and Computer Science \\ University of Illinois at Chicago \\ Chicago, IL 60607
}

April 12, 2013

\begin{abstract}
Grating scattering is a fundamental model in remote sensing, electromagnetics, ocean acoustics, nondestructive testing, and image reconstruction. In this work we examine the problem of detecting the geometric properties of gratings in a two-dimensional acoustic medium where the fields are governed by the Helmholtz equation. Building upon our previous Boundary Perturbation approach (implemented with the Operator Expansions formalism) we derive a new approach which augments this with a new "smoothing" mechanism. With numerical simulations we demonstrate the enhanced stability and accuracy of our new approach which further suggests not only a rigorous proof of convergence, but also a path to generalizing the algorithm to multiple layers, three dimensions, and the full equations of linear elasticity and Maxwell's equations.
\end{abstract}

Keywords: Boundary Perturbations; Operator Expansions; Linear Acoustics; Regularization; Quadratic Optimization.

\section{Introduction}

Grating scattering is a fundamental model in remote sensing [TKS85], electromagnetics [Pet80], ocean acoustics [BL82], nondestructive testing [Shu02], and image reconstruction [NW01]. In a recent paper [MN11a] we devised a Boundary Perturbation technique (based upon the Operator Expansions - OE-[Mil91a, CS93] formalism) for approximating solutions of two related problems in these fields: 1.) The "forward problem" of simulating scattering returns of known incident radiation interacting with a grating of known structure; and 2.) the "inverse problem" of recovering the grating structure given data about both the incident and scattered radiation. In this work we augment and, as we shall see, 
vastly improve our approach to (2.) with the addition of a "smoothing" mechanism similar in spirit (though not identical to) classical Tikhonov regularization [Kre99].

As with the OE method as it was originally designed by Milder [Mil91a, Mil91b, Mil96b, Mil96a] and Milder \& Sharp [MS91, MS92], our new approach is spectrally accurate (i.e., has convergence rates faster than any polynomial order) due to both the analyticity of the scattered fields with respect to boundary perturbation, and the optimal choice of spatial basis functions which arise naturally in the methodology. Our inversion strategy was originally inspired by the work of Nicholls \& Taber [NT08, NT09] on the recovery of topography shape under a layer of an ideal fluid (e.g. the ocean) which also uses the explicit nature of the $\mathrm{OE}$ formulas to great effect.

This contribution is most similar in spirit to the work of Ito \& Reitich [IR99] who considered the same problem in a similar, but not identical, framework. They employed the Field Expansions [BR93a, BR93b, BR93c] method as the underlying "forward solver," and used a regularization to realize greater stability. By contrast, we use the Operator Expansions [Mil91a, CS93] formalism which not only has different numerical properties (see, e.g., [NR01a, NR01b]), but also features formulas where the interface deformation appears explicitly which, as we demonstrate in $\S \S 4.2 \& 4.4$, lead to elegant and easily-derived inversion formulas. In addition, our regularization features a slightly different cost functional to be minimized which leads to a constrained quadratic optimization problem rather than a more general nonlinear one. While this comes at the cost of greater demands placed upon the measurement of the far-field data, it is rewarded with a vastly simplified numerical procedure (the "Null Space Method" of Quadratic Programming [NW06]) which involves inversion of easily precomputed matrices without line searches, derivative calculations, conjugate gradient implementations, nonconvexities, etc.

Other approaches to this problem have, of course, been considered and we refer to Ito \& Reitich's [IR99] paper and the classical texts of Colton \& Kress [CK83, CK98, Kre99] for extensive lists. We mention that most of these are based upon Integral Equation (IE) formulations requiring explicit knowledge of the Green's function. These methods, like our Boundary Perturbation approach, are accurate and efficient as they posit surface unknowns and are spectrally accurate. However, we mention that our current approach is clearly advantaged compared to IE methods in two aspects:

1. For the periodic problems we consider here IE approaches must faithfully compute the periodized Greens function (e.g., via Ewald summation) which is not only technically challenging, but also introduces an additional discretization parameter thereby significantly increasing the computational cost. (See the extensive discussion in [KR09] for a full discussion of these matters together with suggestions to minimize these issues.) Due to the Fourier basis functions utilized by our scheme, these quasiperiodic solutions are computed "by default" at no additional cost.

2. As we mentioned above, the impetus for using the Operator Expansions formalism is due to the fact that the boundary deformations and their powers appear explicitly in this formulation. As with the Field Expansions recursions, the boundary shape appears in the IE formulation in a rather implicit fashion rendering the identification of formulas akin to those in $\S 4$ impossible.

Specific to the simulation of the scattered field from a grating using IE methods, most recently we are aware of the work of Arens \& Grinberg [AG05] on the "Complete Factorization Method" as applied to periodic gratings, the iterative regularization method of Hettlich 
[Het02], and the papers of Bruckner et al [BE05, EHR04, BE03] on the generalization of the Kirsch-Kress optimization method to gratings. In this latter work, [BE05, BE03] focused upon Integral Equation formulations, while [EHR04] modified these approaches to the Finite Element framework. We also point out the work of Kress \& Tran [KT00], Akduman, Kress, \& Yapar [AKY06], Lahcene \& Gaitan [LG11], and the bibliographies of these.

The organization of the paper is as follows: In $\S 2$ we recall the governing equations, including a discussion of relevant unknowns $(\S 2.1)$. In $\S 3$ we discuss the forward problem with relevant expansions presented in $\S 3.1$ followed by brief numerical results in $\S 3.2$. In $\S 4$ we discuss the particulars of the inverse problem and review our previous Boundary Perturbation approach to this problem, specifically we give a formula for the mean depth in $\S 4.1$, a linear approximation for the perturbation shape in $\S \S 4.2 \& 4.3$, and a nonlinearly corrected version in $\S 4.4$. We describe our new, regularized version of this algorithm in $\S 5$, with the principal ideas for a linear algorithm in $\S 5.1$, precise details in $\S 5.3$ (inspired by the procedures for Constrained Quadratic Programming which we recall in $\S 5.2$ ), and a new regularized nonlinear correction given in $\S 5.4$. We close with extensive numerical results in $\S 6$.

\section{Governing Equations}

In this contribution we focus on the problem of recovering the interface of a two-dimensional acoustic medium overlying an impenetrable, periodic solid. Without loss of generality we assume that observations may be made at $y=0$, the interface is "centered" at the (unknown) level $y=\bar{g}<0$, and has (unknown) deviation $g(x)$ such that

$$
\bar{g}+g(x)<0,
$$

see Figure 1.

For simplicity we focus upon the case of a "sound soft" lower material, isonified from above by time-harmonic incident radiation

$$
u^{i}(x, y)=e^{i \alpha x-i \beta y} .
$$

It is well known that the scattered field, $u=u(x, y)$, is governed by the Dirichlet problem [Pet80, MN11a]

$$
\begin{array}{ll}
\Delta u+k^{2} u=0 & y>\bar{g}+g(x) \\
\partial_{y} u-\left(i \beta_{D}\right) u=0 & y=0 \\
u(x, \bar{g}+g(x))=\zeta(x) & \\
u(x+d, y)=e^{i \alpha d} u(x, y), &
\end{array}
$$

where

$$
\zeta(x):=-u^{i}(x, \bar{g}+g(x))=-e^{i \alpha x-i \beta(\bar{g}+g(x))} .
$$

To explain the "Outgoing Wave Condition" (OWC), (2.1b), and introduce important notation, we recall that a solution of (2.1a) \& (2.1d) via separation of variables gives the Rayleigh expansion (see [Pet80], Chapter 1, page 10)

$$
u(x, y)=\sum_{p=-\infty}^{\infty} \hat{\psi}_{p} e^{i \alpha_{p} x+i \beta_{p} y}+\sum_{p=-\infty}^{\infty} \hat{\xi}_{p} e^{i \alpha_{p} x-i \beta_{p} y}, \quad y>\bar{g}+|g|_{L^{\infty}},
$$




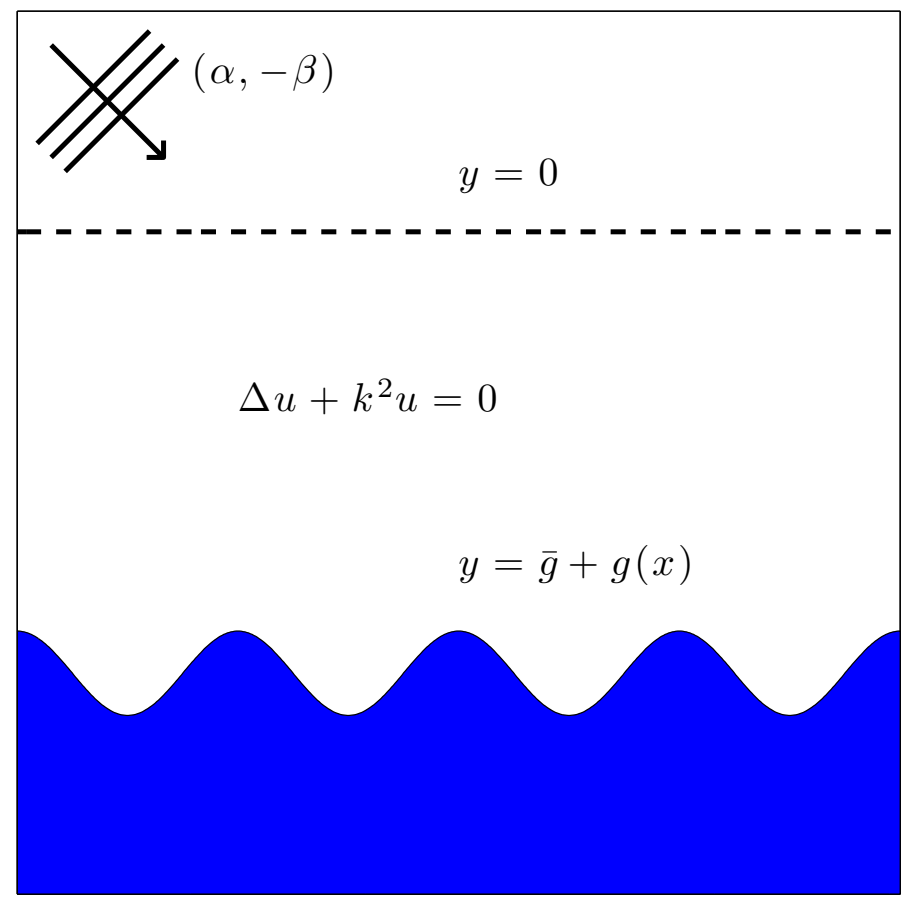

Figure 1: Plot of one-layer configuration with incident radiation $\exp (i \alpha x-i \beta y)$, bottom interface at $y=\bar{g}+g(x)$, and observation plane at $y=0$.

where

$$
\alpha_{p}:=\alpha+\frac{2 \pi p}{d}, \quad \beta_{p}:=\left\{\begin{array}{ll}
\sqrt{k^{2}-\alpha_{p}^{2}} & p \in \mathcal{U} \\
i \sqrt{\alpha_{p}^{2}-k^{2}} & p \notin \mathcal{U}
\end{array},\right.
$$

and the propagating modes are

$$
\mathcal{U}:=\left\{p \in \mathbf{Z} \mid \alpha_{p}^{2}<k^{2}\right\}
$$

The OWC demands that the "downward propagating" coefficients $\hat{\xi}_{p}(p \in \mathcal{U})$ and unbounded modes $\hat{\xi}_{p}(p \notin \mathcal{U})$ be identically zero, while the simple calculation

$$
\partial_{y} u(x, 0)=\sum_{p=-\infty}^{\infty}\left(i \beta_{p}\right) \hat{\psi}_{p} e^{i \alpha_{p} x}=:\left(i \beta_{D}\right) \psi(x)
$$

defines the order-one Fourier multiplier $\left(i \beta_{D}\right)$. As this is simply a matching condition at $y=0$, it is clear that $(2.1 \mathrm{~b})$ enforces the OWC transparently.

Remark. We note that the "sound hard" case can be similarly treated and we present some particulars in Appendix A.

\subsection{Unknowns}

The ideal unknown would be the surface trace

$$
U(x):=u(x, \bar{g}+g(x))
$$


as the governing equations, (2.1), are equivalent to the simple equation

$$
U=\zeta
$$

However, with the inverse problem in mind, it becomes apparent that this is unreasonable as $U$ is posed at the very surface which we do not know. A more promising choice would be

$$
u(x, 0)=: \tilde{u}(x)=\sum_{p=-\infty}^{\infty} \tilde{u}_{p} e^{i \alpha_{p} x},
$$

or, possibly, from the Rayleigh expansion (see [Pet80], Chapter 1, page 10),

$$
B(x):=\sum_{p \in \mathcal{U}} B_{p} e^{i \alpha_{p} x}
$$

We note that since we have chosen $y=0$ as our "measuring plane" we have $\tilde{u}_{p}=B_{p}$, for $p \in \mathcal{U}$. We remark that such quantities are quite reasonable data to measure, as the "far field pattern" for grating scattering is widely regarded as the set of "efficiencies"

$$
e_{p}:=\frac{\beta_{p}}{\beta}\left|B_{p}\right|^{2}=\frac{\beta_{p}}{\beta}\left|\tilde{u}_{p}\right|^{2}, \quad p \in \mathcal{U},
$$

which are effectively (but not identically) $\tilde{u}_{p}$. For later reference we note that, for a lossless material $(\operatorname{Im}\{\beta\}=0)$ there is a principle of conservation of energy [Pet80]:

$$
\sum_{p \in \mathcal{U}} e_{p}=1
$$

so that a widely-accepted diagnostic of convergence (e.g., [Pet80, BR01]) of a numerical scheme is to measure the "energy defect"

$$
\delta:=1-\sum_{p \in \mathcal{U}} e_{p}
$$

\section{The Forward Problem}

Before examining the inverse problem we describe a Boundary Perturbation (BP) method to recover our chosen unknown $\tilde{u}$ from incident radiation, $u^{i}$, and known structure, $\{\bar{g}, g(x)\}$. For this we introduce the "Dirichlet Propagation Operator" (DPO) $P$

$$
P(g)[U(x)]:=\tilde{u}(x)
$$

so that, using (2.2), our governing equation becomes

$$
\tilde{u}(x)=P[U]=P[\zeta(x)]=P(g)[\zeta(x ; g)] .
$$

Our solution procedure is classical regular perturbation theory: If we set $g(x)=\varepsilon f(x)$ and suppose (verifiable a posteriori) that $P$ and $\zeta$ depend analytically upon $\varepsilon$, i.e.

$$
P=P(\varepsilon f)=\sum_{n=0}^{\infty} P_{n}(f) \varepsilon^{n}, \quad \zeta=\zeta(x ; \varepsilon f)=\sum_{n=0}^{\infty} \zeta_{n}(x) \varepsilon^{n},
$$


then we propose an expansion for the unknown

$$
\tilde{u}=\tilde{u}(x ; \varepsilon)=\sum_{n=0}^{\infty} \tilde{u}_{n}(x) \varepsilon^{n},
$$

and, upon insertion of these forms into (3.2), find a formula for the $n$-th correction $\tilde{u}_{n}$ of the form

$$
\tilde{u}_{n}=\sum_{m=0}^{n} P_{n-m}\left[\zeta_{m}\right]
$$

From this we obtain a highly accurate approximation of $\tilde{u}$

$$
\tilde{u}^{N}(x ; \varepsilon):=\sum_{n=0}^{N} \tilde{u}_{n}(x) \varepsilon^{n},
$$

which may be summed directly (Taylor summation) or via numerical analytic continuation methods (Padé summation).

Remark. The limitations of our method are largely determined by the validity of the above expansions. This is characterized by two considerations: First, the smoothness requirements on $f$, and second the size of the perturbation allowed (i.e., the magnitude of $\varepsilon$ ). Regarding each of these we refer the interested reader to the extensive work, both theoretical and numerical, on these questions in connection to Laplace's [NR01a, NR01b, NR03] and Helmholtz's equation [NR04a, NR04b]. In these it is shown that the expansions are valid for quite rough profiles (twice continuously differentiable via elementary methods, or Hölder of index $1+\theta, \theta>0$, and even Lipschitz continuous via more sophisticated techniques [HN05]). Additionally, using analytic continuation techniques the expansions are strongly convergent for real perturbations of arbitrary size, up to physical obstruction (e.g., intersection of layer interfaces) [NR03, HN10].

\subsection{Expansions}

To enable the perturbation strategy above to succeed, the data, $\zeta$, and boundary operator, $P$, must be expanded in Taylor series. These effectively appear in previous publications (see, e.g., [MN11a]), but we briefly rederive them here for completeness. For the data, if we set $g(x)=\varepsilon f(x)$ in $(2.1 \mathrm{e})$ then

$$
\zeta(x ; \varepsilon)=-e^{i \alpha x-i \beta(\bar{g}+\varepsilon f)}=-e^{i \alpha x-i \beta \bar{g}} \sum_{n=0}^{\infty}(-i \beta)^{n} F_{n} \varepsilon^{n},
$$

where $F_{n}(x):=f(x)^{n} / n$ !. Thus

$$
\zeta_{n}(x)=-(-i \beta)^{n} e^{-i \beta \bar{g}} F_{n}(x) e^{i \alpha x} .
$$

Regarding the operator $P$ we appeal to the method of Operator Expansions [Mil91a, CS93] and seek formulas for the action of $P_{n}$ on basis functions, in this case the complex exponentials $\exp \left(i \alpha_{p} x\right)$. Consider the following quasiperiodic solution of the Helmholtz equation satisfying the OWC (i.e., satisfying (2.1a), (2.1b), and (2.1d))

$$
U_{p}(x, y):=e^{i \alpha_{p} x+i \beta_{p}(y-\bar{g})}
$$


and insert this into the definition of $P,(3.1)$ :

$$
P(g)\left[e^{i \alpha_{p} x+i \beta_{p} g}\right]=P(g)\left[U_{p}(x, \bar{g}+g)\right]=\tilde{U}_{p}(x)=U_{p}(x, 0)=e^{i \alpha_{p} x-i \beta_{p} \bar{g}} .
$$

Setting $g=\varepsilon f$ and expanding in Taylor series results in

$$
\left(\sum_{n=0}^{\infty} P_{n}(f) \varepsilon^{n}\right)\left[\sum_{m=0}^{\infty}\left(i \beta_{p}\right)^{m} F_{m} \varepsilon^{m} e^{i \alpha_{p} x}\right]=e^{-i \beta_{p} \bar{g}} e^{i \alpha_{p} x}
$$

At order zero

$$
P_{0}\left[e^{i \alpha_{p} x}\right]=e^{-i \beta_{p} \bar{g}} e^{i \alpha_{p} x},
$$

and upon expanding a typical $\left(L^{2}\right)$ function in its Fourier series

$$
\psi(x)=\sum_{p=-\infty}^{\infty} \hat{\psi}_{p} e^{i \alpha_{p} x}
$$

we find a formula for $P_{0}$ applied to $\psi$ :

$$
P_{0}[\psi]=P_{0}\left[\sum_{p=-\infty}^{\infty} \hat{\psi}_{p} e^{i \alpha_{p} x}\right]=\sum_{p=-\infty}^{\infty} \hat{\psi}_{p} P_{0}\left[e^{i \alpha_{p} x}\right]=\sum_{p=-\infty}^{\infty} \hat{\psi}_{p} e^{-i \beta_{p} \bar{g}} e^{i \alpha_{p} x}=: e^{-i \beta_{D} \bar{g}} \psi
$$

which defines the Fourier multiplier $\exp \left(-i \beta_{D} \bar{g}\right)$.

At order $n>0(3.3)$ yields

$$
\sum_{m=0}^{n} P_{m}(f)\left[\left(i \beta_{p}\right)^{n-m} F_{n-m} e^{i \alpha_{p} x}\right]=0,
$$

which, upon solving for $P_{n}$, leads to

$$
P_{n}(f)\left[e^{i \alpha_{p} x}\right]=-\sum_{m=0}^{n-1} P_{m}(f)\left[\left(i \beta_{p}\right)^{n-m} F_{n-m} e^{i \alpha_{p} x}\right] .
$$

Once again appealing to the Fourier series representation of the function $\psi$ we find

$$
P_{n}(f)[\psi]=-\sum_{m=0}^{n-1} P_{m}(f)\left[F_{n-m}\left(i \beta_{D}\right)^{n-m} \psi\right] .
$$

\subsection{Brief Numerical Results: Forward Problem}

This numerical procedure is very much in the spirit of the work of Milder [Mil91a, Mil91b, Mil96b, Mil96a], Milder \& Sharp [MS91, MS92], Kaczkowski \& Thorsos [KT94], and Nicholls \& Reitich [NR04a, NR04b, NR08] (see also Voronivich's reference [Vor99]) and is not a significant departure from these. However, to give us confidence in the "forward data" which this method can produce, we report on a series of numerical simulations.

We consider the following physical parameters

$$
\alpha=0, \quad \beta=5.5, \quad d=2 \pi, \quad \bar{g}=-1.5,
$$


so that $k=\sqrt{\alpha^{2}+\beta^{2}}=\beta=5.5$, and an analytic interface profile with Fourier coefficients given by

$$
\hat{f}_{p}=\left\{\begin{array}{ll}
\frac{1}{2}(2 \rho)^{(|p|-1) /(R-1)} & p \neq 0 \\
0 & p=0
\end{array} .\right.
$$

We make this particular choice as it has mean zero $\left(\hat{f}_{0}=0\right)$, shares some height/slope characteristics of the cosine function (since, as with the cosine, $\hat{f}_{1}=\hat{f}_{-1}=1 / 2$ ), and one can specify the value of the $R$-th Fourier coefficient as $\rho$. To get a more concrete feel for this profile, recall [CHQZ88] that if a function has Fourier coefficients

$$
\hat{h}_{p}=\left\{\begin{array}{ll}
C B^{-|p|} & p \neq 0 \\
0 & p=0
\end{array},\right.
$$

where $B>1$, then

$$
h(x)=C\left(\frac{2 B \cos (x)-2}{B^{2}+1-2 B \cos (x)}\right) .
$$

A brief proof comes from the elementary calculation:

$$
\begin{aligned}
h(x) & =\sum_{p=1}^{\infty} C B^{-p} e^{i p x}+\sum_{p=1}^{\infty} C B^{-p} e^{-i p x} \\
& =C\left(\sum_{p=1}^{\infty}\left(B^{-1} e^{i x}\right)^{p}+\sum_{p=1}^{\infty}\left(B^{-1} e^{-i x}\right)^{p}\right) \\
& =C\left(\frac{B^{-1} e^{i x}}{1-B^{-1} e^{i x}}+\frac{B^{-1} e^{-i x}}{1-B^{-1} e^{-i x}}\right) \\
& =C\left(\frac{e^{i x}}{B-e^{i x}}+\frac{e^{-i x}}{B-e^{-i x}}\right) \\
& =C\left(\frac{\left(B e^{i x}-1\right)+\left(B e^{-i x}-1\right)}{B^{2}+1-B e^{i x}-B e^{-i x}}\right) \\
& =C\left(\frac{2 B \cos (x)-2}{B^{2}+1-2 B \cos (x)}\right) .
\end{aligned}
$$

In our case

$$
B=(2 \rho)^{-1 /(R-1)}, \quad C=\frac{1}{2} B
$$

so that

$$
f(x)=C\left(\frac{2 B \cos (x)-2}{B^{2}+1-2 B \cos (x)}\right)=\frac{B^{2} \cos (x)-B}{B^{2}+1-2 B \cos (x)} .
$$

For $(\varepsilon, R)$ values $(0.01,10),(0.03,20)$, and $(0.05,30)$ with $\rho=10^{-16}$, we display in Figure 2 the energy defect $\delta=\delta(\varepsilon)$ versus perturbation order $N$. In these results we truncate all Fourier series beyond $\pm N_{x} / 2$ coefficients, and have selected $N_{x}=128$. The perturbation series are summed via classical Padé approximation [BGM96] as we have in previous work [NR01b, NR03, MN11b]. In all three cases, we see the rapid and reliable convergence of our approach for this analytic yet non-trivial interface. 


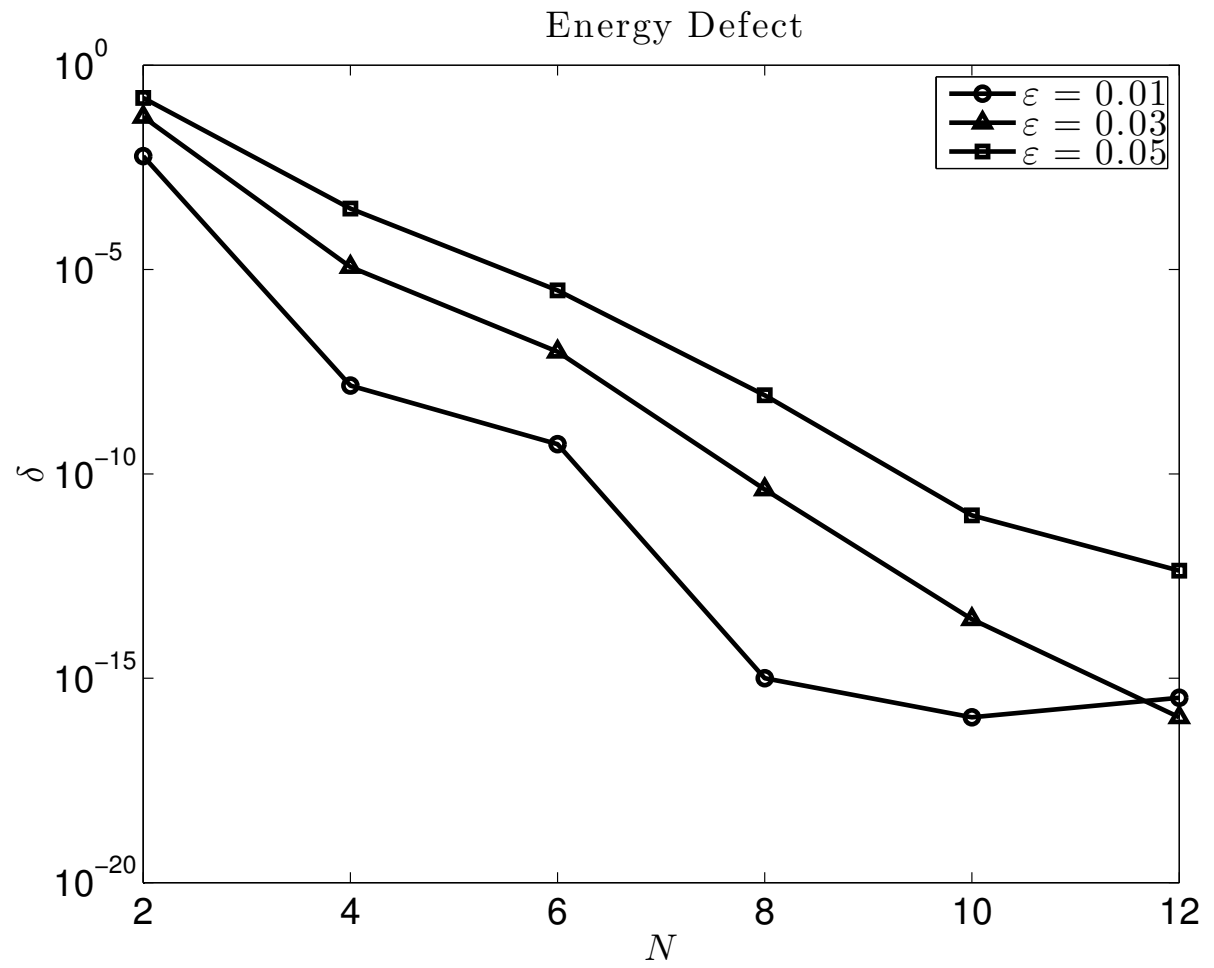

Figure 2: Energy defect, $\delta$, summed via Padé algorithm versus number of perturbation orders retained, $N$. Physical parameters: $\alpha=0.0, \beta=5.5, d=2 \pi, \bar{g}=-1.5$; profile parameters: $\varepsilon=0.01,0.03,0.05, R=10, \rho=10^{-16}$; numerical parameter: $N_{x}=128$. 


\section{The Inverse Problem}

We now adopt the point of view that the problem knowns are the incident wavenumber $(\alpha, \beta)$ and some scattered far-field data. For these we request a collection of Fourier coefficients $\left\{\hat{\eta}_{p}\right\}$ such that

$$
\eta(x):=\sum_{p \in \mathcal{P}} \hat{\eta}_{p} e^{i \alpha_{p} x} \approx \tilde{u}(x) .
$$

We leave the set of modes $\mathcal{P}$ unspecified but remark that we can certainly expect the propagating modes, $\mathcal{U} \subset \mathcal{P}$, and any (reliable) additional evanescent modes, $p \notin \mathcal{U}$, will simply add to the accuracy of our method.

\subsection{Order Zero Approximation: Formula for $\bar{g}$}

We recall that our governing equation, (3.2), is

$$
\tilde{u}=P[\zeta]=P(\bar{g}, g)[\zeta(x ; \bar{g}, g)]
$$

and, making the expansions we utilized with such success in $\S 3$, we write

$$
\tilde{u}=P_{0}\left[\zeta_{0}\right]+\mathcal{O}(g) .
$$

Ignoring terms of order $g$ and higher we have the approximation

$$
\tilde{u} \approx P_{0}\left[\zeta_{0}\right] .
$$

If we now enforce both $\tilde{u}=\eta$ and $\tilde{u}=P_{0}\left[\zeta_{0}\right]$, and insert the forms for $P_{0}$ and $\zeta_{0}$ which we derived in $\S 3.1$, we find

$$
\begin{aligned}
\sum_{p=-\infty}^{\infty} \hat{\eta}_{p} e^{i \alpha_{p} x} & =\eta=\tilde{u}=P_{0}\left[\zeta_{0}\right]=e^{-i \beta_{D} \bar{g}}\left[-e^{-i \beta \bar{g}} e^{i \alpha x}\right]=e^{-i \beta_{D} \bar{g}}\left[\sum_{p=-\infty}^{\infty}-e^{-i \beta \bar{g}} e^{i \alpha_{p} x} \delta_{p, 0}\right] \\
& =\sum_{p=-\infty}^{\infty} e^{-i \beta_{p} \bar{g}}\left[-e^{-i \beta \bar{g}} e^{i \alpha_{p} x} \delta_{p, 0}\right]=-e^{-i \beta \bar{g}} e^{-i \beta_{0} \bar{g}} e^{i \alpha_{0} x}=-e^{-2 i \beta \bar{g}} e^{i \alpha x},
\end{aligned}
$$

where $\delta_{p, q}$ is the Kronecker delta function, and we recall that $\alpha_{0}=\alpha$ and $\beta_{0}=\beta$. Clearly this approximation is inaccurate as it is most likely that $\hat{\eta}_{p} \neq 0$ for $p \neq 0$. However, supposing that both $\left|\hat{\eta}_{p}\right| \ll 1$ for $p \neq 0$, and that $\left|\hat{\eta}_{0}\right| \approx 1$, by equating arguments we find

$$
\operatorname{Arg}\left\{\hat{\eta}_{0}\right\}=-2 \beta \bar{g}+2 \pi m+\pi
$$

for any integer $m$. The ill-posedness of our inverse problem is on full display in this equation as there are an infinite number of solutions. However, we can make an educated selection and simply pick

$$
\bar{g}=\frac{-\operatorname{Arg}\left\{\hat{\eta}_{0}\right\}+2 \pi \bar{m}+\pi}{2 \beta}
$$

for the $\bar{m}$ which selects the shallowest layer (i.e., the negative $\bar{g}$ of smallest magnitude). 


\subsection{Linear Approximation (LA): Formula for $g$}

Motivated by our previous work [MN11a], we write the governing equation (3.2) as

$$
\tilde{u}=P_{0}\left[\zeta_{0}\right]+\left\{P_{1}(\cdot)\left[\zeta_{0}\right]+P_{0}\left[\zeta_{1}(\cdot)\right]\right\}[g]+\mathcal{O}\left(g^{2}\right) .
$$

Ignoring terms of order $g^{2}$ we consider

$$
\tilde{u}=b+M[g]
$$

where

$$
\begin{aligned}
b & :=P_{0}\left[\zeta_{0}\right], \\
M & :=\left\{P_{1}(\cdot)\left[\zeta_{0}\right]+P_{0}\left[\zeta_{1}(\cdot)\right]\right\} .
\end{aligned}
$$

Simply setting $\tilde{u}=\eta$, so that we match the far-field data exactly, we find $g$ from the "Linear Approximation" (LA)

$$
M[g]=\eta-b,
$$

which is essentially the "Linear Model" in [MN11a] adapted to the one-layer case.

\subsection{The Operator $M$}

The ill-conditioning of the inverse problem becomes apparent in this formula with a careful analysis of $M$. Recall that, defining $\phi(x):=\exp (i(-\beta \bar{g}+\alpha x))$,

$$
P_{0}[\psi]=e^{-i \beta_{D} \bar{g}}[\psi], \quad \zeta_{0}(x)=-\phi(x),
$$

and that

$$
P_{1}(g)[\psi]=-P_{0}\left[g\left\{\left(i \beta_{D}\right)[\psi]\right\}\right], \quad \zeta_{1}(x ; g)=(i \beta) g(x) \phi(x)
$$

so that

$$
\begin{aligned}
M[g] & =P_{1}(g)\left[\zeta_{0}(x)\right]+P_{0}\left[\zeta_{1}(x ; g)\right] \\
& =-P_{0}\left[g\left\{\left(i \beta_{D}\right)[-\phi]\right\}\right]+P_{0}[(i \beta) g \phi] \\
& =P_{0}\left[g\left\{\left(i \beta_{D}\right)[\phi]\right\}+(i \beta) g \phi\right] .
\end{aligned}
$$

As we saw in (4.1), it is not difficult to show that

$$
\left(i \beta_{D}\right) \phi=\left(i \beta_{D}\right) e^{-i \beta \bar{g}} e^{i \alpha x}=e^{-i \beta \bar{g}}\left(i \beta_{D}\right) \sum_{p=-\infty}^{\infty} \delta_{0, p} e^{i \alpha_{p} x}=e^{-i \beta \bar{g}} \sum_{p=-\infty}^{\infty}\left(i \beta_{p}\right) \delta_{0, p} e^{i \alpha_{p} x}=(i \beta) \phi,
$$

so that

$$
M[g]=P_{0}[g(i \beta) \phi+(i \beta) g \phi]=(2 i \beta) P_{0}[\phi g] .
$$

Therefore, $M$ is essentially the exponentially smoothing operator $P_{0}$ (multiplication by $\phi$ simply delivers $(\phi g)$ which is quasiperiodic versus the periodic function $g$ ) with inverse

$$
P_{0}^{-1}[\psi(x)]=\sum_{p=-\infty}^{\infty} e^{i \beta_{p} \bar{g}} \hat{\psi}_{p} e^{i \alpha_{p} x}
$$

and we point out that, since $\bar{g}<0$, this operator will exponentially amplify the evanescent wavenumbers, $p \notin \mathcal{U}$, giving a severely ill-posed operator. 


\subsection{Nonlinear Approximation (NLA): Iteration Scheme for $g$}

Continuing with the program of [MN11a] we write our governing equation (3.2) as

$$
\tilde{u}=P_{0}\left[\zeta_{0}\right]+\left\{P_{1}(\cdot)\left[\zeta_{0}\right]+P_{0}\left[\zeta_{1}(\cdot)\right]\right\}[g]+R^{\left(N_{i}\right)}(g)+\mathcal{O}\left(g^{N_{i}+1}\right),
$$

for an approximation order $N_{i}$ where

$$
R^{\left(N_{i}\right)}(g):=\sum_{n=2}^{N_{i}} \sum_{m=0}^{n} P_{m}(g)\left[\zeta_{n-m}(x ; g)\right] .
$$

Ignoring terms of order $g^{N_{i}+1}$ we consider

$$
\tilde{u}=b+M[g]+R^{\left(N_{i}\right)}(g)
$$

and, after setting $\tilde{u}=\eta$, we set up the "Nonlinear Approximation" (NLA) iteration for the approximations $g^{(k+1)}$ :

$$
M\left[g^{(k+1)}\right]=\eta-b-R^{\left(N_{i}\right)}\left(g^{(k)}\right),
$$

which is the "Nonlinear Model" in [MN11a] adapted to the one-layer case. We use the solution of the LA algorithm, (4.5), as the initial guess $g^{(0)}$.

\section{Regularizing the Inverse Problem}

A standard technique from the theory of inverse problems [Kre99] for regularizing ill-posed problems of the form

$$
A \varphi=f
$$

is to minimize the quantity

$$
\|A \varphi-f\|^{2}+\tau\|\varphi\|^{2}, \quad \tau>0
$$

which, if $A$ is compact, has unique solution, $\varphi^{\tau}$, satisfying

$$
\left(\tau I+A^{*} A\right) \varphi^{\tau}=A^{*} f
$$

This Tikhonov Regularization technique [Kre99] could, of course, be applied to our LA scheme, (4.5), where we set $\tilde{u}=\eta$ (i.e. that we measure the far-field exactly) and $\varphi=g$.

However, we choose to pursue a slightly different approach in which we allow a "defect" in the far-field measurement, $(\tilde{u} \neq \eta)$, view the function pair

$$
X:=(\tilde{u}, g)^{T}
$$

as our unknown, and seek minimizers for the form

$$
\tilde{q}(\tilde{u}, g):=\frac{1}{2}\|\tilde{u}-\eta\|_{L^{2}}^{2}+\frac{\tau}{2}\left(\|g\|_{L^{2}}^{2}+\left\|\partial_{x} g\right\|_{L^{2}}^{2}\right),
$$

for $\tau>0$. We will constrain our search by demanding that the pair $(\tilde{u}, g)$ satisfy the governing equations (3.2), and note that $\tilde{q}$ penalizes both defect between actual and experimental 
far-field pattern, and oscillations in $g$. We do not change the set of minimizers by simply subtracting the constant term $\frac{1}{2}\|\eta\|_{L^{2}}^{2}$, and thus we focus on the quantity

$$
\begin{aligned}
q(\tilde{u}, g) & :=\frac{1}{2}\|\tilde{u}-\eta\|_{L^{2}}^{2}-\frac{1}{2}\|\eta\|_{L^{2}}^{2}+\frac{\tau}{2}\left(\|g\|_{L^{2}}^{2}+\left\|\partial_{x} g\right\|_{L^{2}}^{2}\right) \\
& =\frac{1}{2}\langle\tilde{u}, \tilde{u}\rangle-\operatorname{Re}\{\langle\eta, \tilde{u}\rangle\}+\frac{\tau}{2}\left(\langle g, g\rangle+\left\langle\partial_{x} g, \partial_{x} g\right\rangle\right) \\
& =\frac{1}{2}\langle\tilde{u}, \tilde{u}\rangle-\operatorname{Re}\{\langle\eta, \tilde{u}\rangle\}+\frac{\tau}{2}\left\langle g,\left(I-\partial_{x}^{2}\right) g\right\rangle,
\end{aligned}
$$

where

$$
\langle f, g\rangle:=\int_{0}^{d} f(x) \overline{g(x)} d x,
$$

and we have used integration by parts. Introducing the notation

$$
Q:=\left(\begin{array}{cc}
I & 0 \\
0 & \tau\left(I-\partial_{x}^{2}\right)
\end{array}\right), \quad c:=\left(\begin{array}{l}
\eta \\
0
\end{array}\right)
$$

we can write $q$ as

$$
q(X)=\frac{1}{2}\langle X, Q X\rangle-\operatorname{Re}\{\langle c, X\rangle\}
$$

\subsection{Regularized Linear Approximation (RLA): Formula for $g$}

Recalling our governing equation, (3.2), we define a constraint function

$$
B(\tilde{u}, g):=\tilde{u}-P(g) \zeta(g),
$$

and, from (4.3), observe that

$$
B(\tilde{u}, g)=\tilde{u}-P_{0} \zeta_{0}-\left\{P_{1}(\cdot)\left[\zeta_{0}\right]+P_{0}\left[\zeta_{1}(\cdot)\right]\right\}[g]+\mathcal{O}\left(g^{2}\right),
$$

so that, recalling (4.4), making the definition

$$
B^{(1)}(\tilde{u}, g):=\tilde{u}-b-M[g]
$$

seems reasonable. If we set

$$
A:=(I-M),
$$

then

$$
B^{(1)}(\tilde{u}, g)=A X-b .
$$

We consider our "Regularized Linear Approximation" (RLA) as finding the $X$ satisfying the constrained quadratic optimization problem:

$$
\begin{aligned}
\min _{X} q(X)= & \min _{X}\left\{\frac{1}{2}\langle X, Q X\rangle-\operatorname{Re}\{\langle c, X\rangle\}\right\} \\
& \text { such that } A X=b .
\end{aligned}
$$




\subsection{Constrained Quadratic Optimization}

To explain our numerical procedure for solving (5.4) we recall the procedure for the corresponding problem in finite dimensions [NW06]. To avoid confusion with our notation for the infinite-dimensional problem, yet be suggestive with the details of our algorithm, we denote matrices with bold-face and vectors with an over-arrow, while using the same letter for corresponding quantities (e.g., functions and vectors, operators and matrices). Consider the functional

$$
q(\vec{x}):=\frac{1}{2} \vec{x}^{*} \mathbf{Q} \vec{x}-\vec{c}^{*} \vec{x}
$$

where $\mathbf{Q} \in \mathbf{C}^{n \times n}$ is Hermitian and positive definite, $\vec{x}, \vec{c} \in \mathbf{C}^{n}$, and $*$ denotes (conjugate) transpose. We seek to minimize this subject to the set of linear constraints

$$
\vec{B}(\vec{x}):=\mathbf{A} \vec{x}-\vec{b}=0,
$$

where $\mathbf{A} \in \mathbf{C}^{m \times n}, \vec{b} \in \mathbf{C}^{m}, m \leq n$, and $\mathbf{A}$ has full row rank $m$. It is well known [NW06] that the unique solution of this problem can be found by the method of Lagrange multipliers in the following way. Consider the Lagrangian

$$
\mathcal{L}(x):=q(\vec{x})-\vec{\lambda}^{*} \vec{B}(\vec{x}),
$$

with Lagrange multiplier $\vec{\lambda} \in \mathbf{C}^{m}$. The first-order condition that the function $q$ be minimized is that the gradient of the Lagrangian be zero:

$$
0=\nabla_{x} \mathcal{L}(\vec{x})=\mathbf{Q} \vec{x}-\vec{c}-\mathbf{A}^{*} \vec{\lambda} .
$$

Coupling this to the constraint $\vec{B}(\vec{x})=0$ gives the Karush-Kuhn-Tucker (KKT) equations

$$
\left(\begin{array}{cc}
\mathbf{Q} & -\mathbf{A}^{*} \\
\mathbf{A} & 0
\end{array}\right)\left(\begin{array}{l}
\vec{x} \\
\vec{\lambda}
\end{array}\right)=\left(\begin{array}{l}
\vec{c} \\
\vec{b}
\end{array}\right)
$$

To solve this system of equations we have two options [NW06]: Schur complements if $\mathbf{Q}$ is invertible (and easy to apply), and the "Null Space Method" if the null space of $\mathbf{A}$ is known. With a view to future applications with multiply layered media where $\mathbf{Q}$ may not be invertible, we advocate the latter approach and assume that we can find the matrix $\mathbf{Z} \in \mathbf{C}^{n \times(n-m)}$ containing, as columns, $(n-m)$ linearly independent members of $\mathcal{N}(\mathbf{A})$ so that

$$
\mathbf{A Z}=0 .
$$

Now, if we can easily find $\mathbf{Y} \in \mathbf{C}^{n \times m}$ such that the matrix $(\mathbf{Y} \mid \mathbf{Z})$ is non-singular then we express $\vec{x}$ as

$$
\vec{x}=\mathbf{Y} \vec{y}+\mathbf{Z} \vec{z},
$$

a portion in the null space of $\mathbf{A}$ and "another part." Now, inserting this into the second equation of (5.5), and using $\mathbf{A Z}=0$, we recover

$$
(\mathbf{A Y}) \vec{y}=\vec{b}
$$

and it is not difficult to show that (AY) is invertible [NW06]. Now, the first equation of (5.5) reads

$$
\mathbf{Q}[\mathbf{Y} \vec{y}+\mathbf{Z} \vec{z}]-\mathbf{A}^{*} \vec{\lambda}=\vec{c} .
$$


Left-multiplying by $\mathbf{Z}^{*}$ and recognizing $\mathbf{Z}^{*} \mathbf{A}^{*}=(\mathbf{A Z})^{*}=0$ delivers

$$
\left(\mathbf{Z}^{*} \mathbf{Q Z}\right) \vec{z}=\mathbf{Z}^{*}[\vec{c}-\mathbf{Q Y} \vec{y}]
$$

where $\mathbf{K}:=\left(\mathbf{Z}^{*} \mathbf{Q Z}\right) \in \mathbf{C}^{(n-m) \times(n-m)}$ can be shown to be invertible [NW06] and $\vec{y}$ is now known. Finally, left-multiplying the first equation in (5.5) by $\mathbf{Y}^{*}$ gives

$$
(\mathbf{A Y})^{*} \vec{\lambda}=\mathbf{Y}^{*}[\mathbf{Q}(\mathbf{Y} \vec{y}+\mathbf{Z} \vec{z})-\vec{c}]=\mathbf{Y}^{*}[\mathbf{Q} \vec{x}-\vec{c}],
$$

where $(\mathbf{A Y})^{*}$ is nonsingular as $(\mathbf{A Y})$ is. Summarizing, we solve, in order, the following

$$
\begin{aligned}
& (\mathbf{A Y}) \vec{y}=\vec{b} \\
& \mathbf{K} \vec{z}=\mathbf{Z}^{*}[\vec{c}-\mathbf{Q Y} \vec{y}] \\
& (\mathbf{A Y})^{*} \vec{\lambda}=\mathbf{Y}^{*}[\mathbf{Q} \vec{x}-\vec{c}],
\end{aligned}
$$

where each of the operators $(\mathbf{A Y}), \mathbf{K}$, and $(\mathbf{A Y})^{*}$ is invertible.

\subsection{Regularized Linear Algorithm: Formula}

We now solve (5.4) by minimizing the function $q$, (5.2), using the "Null Space Method," (5.6), with $Q$ and $c$ given in (5.1), and $A$ given in (5.3). Inspired by the developments of the previous section we define the following operator-valued matrices

$$
Z=\left(\begin{array}{c}
M \\
I
\end{array}\right), \quad Y=\left(\begin{array}{l}
I \\
0
\end{array}\right)
$$

and find:

$$
A Y=I, \quad(A Y)^{*}=I, \quad K=M^{*} M+\tau\left(I-\partial_{x}^{2}\right) .
$$

The same manipulations we displayed in the finite dimensional case can be performed again and, if we seek a solution $x=Y y+Z z$, we find, c.f. (5.6a),

$$
y=b .
$$

Using this calculation we discover that

$$
Z^{*}[c-Q Y y]=M^{*}[\eta-b]
$$

so that, c.f. $(5.6 \mathrm{~b})$,

$$
\left[M^{*} M+\tau\left(I-\partial_{x}^{2}\right)\right] z=M^{*}[\eta-b] .
$$

Having found $y$ and $z$ we can form $X=(\tilde{u}, g)^{T}$ and recover, c.f. (5.6c), that

$$
\lambda=Y^{*}[Q x-c]=\tilde{u}-\eta .
$$

Vanishing Smoothing Limit: In the limit $\tau \rightarrow 0$, we find

$$
y=b, \quad M^{*} M z=M^{*}[\eta-b], \quad \lambda=\tilde{u}-\eta,
$$

so that $z$ is the least squares solution of the ill-posed equation $M z=\eta-b$. If $M^{-1}$ existed then

$$
z=M^{-1}[\eta-b]
$$

so that

$\left(\begin{array}{c}\tilde{u} \\ g\end{array}\right)=X=Y y+Z z=Y b+Z M^{-1}[\eta-b]=\left(\begin{array}{c}I \\ 0\end{array}\right) b+\left(\begin{array}{c}M \\ I\end{array}\right) M^{-1}[\eta-b]=\left(\begin{array}{c}\eta \\ M^{-1}[\eta-b]\end{array}\right)$,

which is simply (4.5). 


\subsection{Regularized Nonlinear Approximation (RNLA): Iteration Scheme for $g$}

In light of the success we had in our nonlinear versus linear approximations (NLA versus LA) $[\mathrm{MN11a}]$, we mimic those ideas in the setting of the regularization outlined above, (5.4). Following that development we note that, from (4.6),

$$
B(\tilde{u}, g)=b+M[g]+R^{\left(N_{i}\right)}(g)+\mathcal{O}\left(g^{N+i+1}\right)
$$

which inspires

$$
B^{\left(N_{i}\right)}(\tilde{u}, g):=\tilde{u}-b-M[g]-R^{\left(N_{i}\right)}(g) .
$$

Using (5.3) once again, we find

$$
B^{\left(N_{i}\right)}(\tilde{u}, g)=A X-b-R^{\left(N_{i}\right)}(g)
$$

and consider the iterative "Regularized Nonlinear Linear Approximation" (RNLA) as finding the $X^{(k+1)}$ satisfying the constrained quadratic optimization problem:

$$
\begin{aligned}
\min _{X^{(k+1)}} q\left(X^{(k+1)}\right) & =\min _{X^{(k+1)}}\left\{\frac{1}{2}\left\langle X^{(k+1)}, Q X^{(k+1)}\right\rangle-\operatorname{Re}\left\{\left\langle c, X^{(k+1)}\right\rangle\right\}\right\} \\
& \text { such that } A X^{(k+1)}=b+R^{\left(N_{i}\right)}\left(g^{(k)}\right)
\end{aligned}
$$

where we select $X^{(0)}$ as the solution of the RLA, (5.4).

\section{Numerical Results: Inverse Problem}

We now demonstrate the convergence behavior of these four algorithms: The Linear Algorithm (LA) (4.5), the Nonlinear Algorithm (NLA) (4.7), the Regularized Linear Algorithm (RLA) (5.4), and the Regularized Nonlinear Algorithm (RNLA) (5.7). Once again we select the physical parameters (3.6):

$$
\alpha=0, \quad \beta=5.5, \quad d=2 \pi, \quad \bar{g}=-1.5,
$$

the analytic profile, $(3.8)$, with $\rho=10^{-16}$ and choices $(\varepsilon, R)=(0.01,10),(0.03,20),(0.05,30)$. The far-field data that we use to generate our approximations are generated by our forward algorithm (3.2) with $N_{x}=128$ and $N=12$. We point out that by doing this we are committing the "inverse crime" [CK98] of using a philosophically similar method to generate data for the inversion strategy. Colton \& Kress [CK98] (page 133, $\S 5.3$ ) point out that by doing this it is unsurprising that "... the surface ... is recovered pretty well." With this in mind, in $\S 6.1$ we add noise to the synthetically generated input data to not only address this criticism but also more realistically simulate "real world" data.

Recalling the definition of the $L^{\infty}$ norm,

$$
|f|_{L^{\infty}}:=\sup _{0<x<d}|f(x)|
$$

where the essential supremum is intended, in Figure 3 we display a plot of relative $L^{\infty}$ error compared with the exact solution versus the smoothing parameter $\tau$ in the case $(\varepsilon=$ $0.01, R=10)$. We note that for the LA and NLA methods, as there is no smoothing, we simply plot the error as a constant function. Furthermore, the NLA line does not appear 


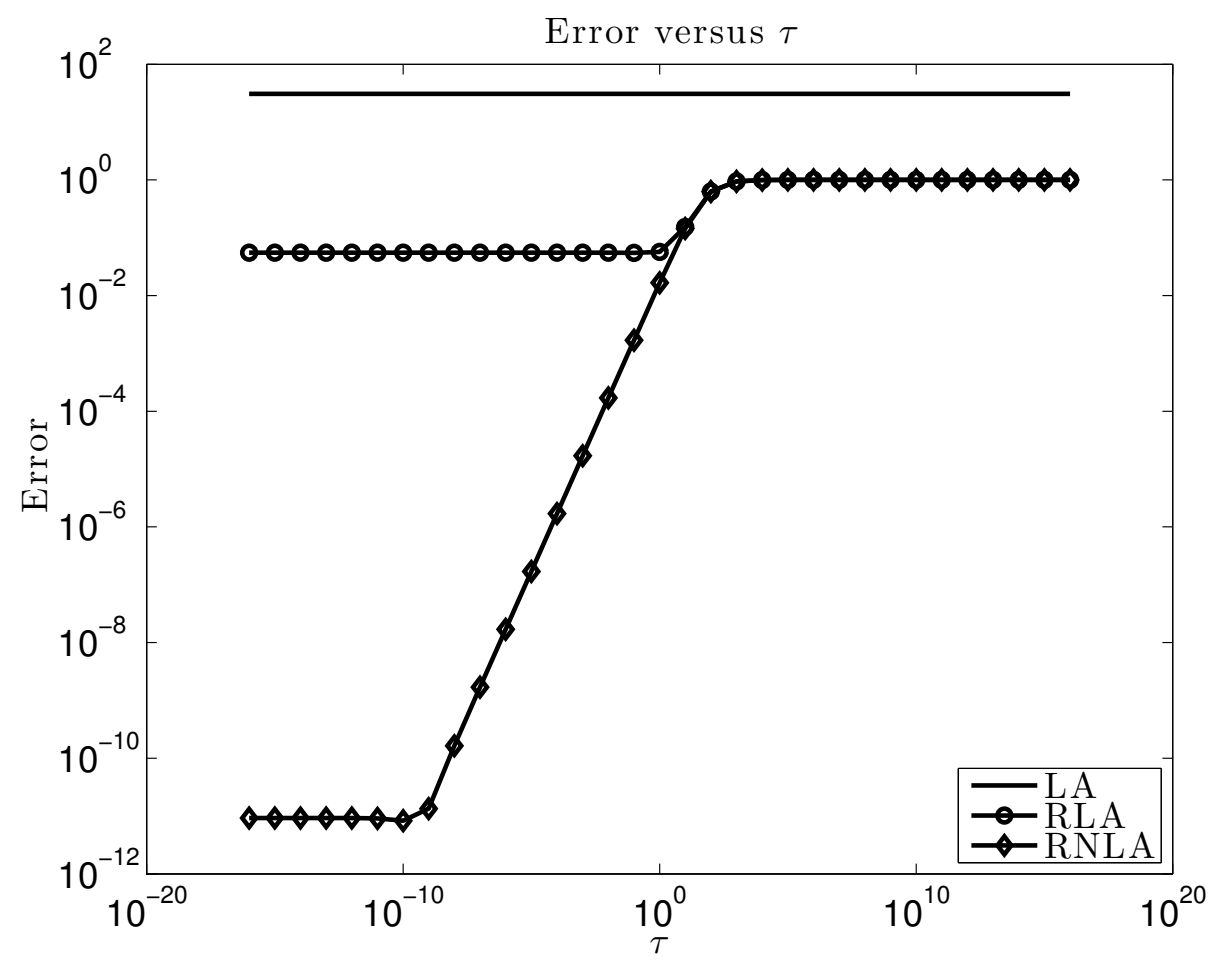

Figure 3: Plot of relative $L^{\infty}$ error in reconstructed solution (compared to the exact solution) versus the regularization parameter $\tau$ for the LA, RLA, and RNLA algorithms. Physical parameters: $\alpha=0.0, \beta=5.5, d=2 \pi, \bar{g}=-1.5$; profile parameters: $\varepsilon=0.01, R=10$, $\rho=10^{-16}$; numerical parameters: $N_{x}=128, N_{i}=8$.

as the algorithm diverges for this discretization due to ill-conditioning. In the less-resolved case $N_{x}=32$ we can recover excellent convergence for this method, c.f. [MN11a]; see Figure 4. In Figure 3 we notice the extremely beneficial gains in accuracy which can be realized with both the regularizer (three orders of magnitude improvement for the Linear Algorithm), and the nonlinear correction (over ten more orders of magnitude improvement). We point out that in this and all subsequent simulations, if $\tau$ is chosen sufficiently large then the recovered solution is simply $g(x) \equiv 0$ (resulting in $100 \%$ error).

We repeat these calculations for $(\varepsilon=0.03, R=20)$ and $(\varepsilon=0.05, R=30)$ and display the results in Figures 5 and 6, respectively. Once again, we notice the extremely beneficial effects of using both the regularizer (three orders of magnitude improvement for the Linear Algorithm), and the nonlinear correction (seven or even nine orders of magnitude further improvement).

Remark. Of course the key consideration in all of this is the choice of the regularization parameter $\tau$. This is a subtle and difficult question, and sources like Colton \& Kress [CK98] (page $87, \S 4.2$ ) give some guidance, however, as most must admit "... quite often the only choice for selecting the regularization will be trial and error ..." [CK98].

\subsection{Reconstruction in the Presence of Noise}

As the goal of our work is to apply these algorithms to data collected either in the laboratory or the field, their stability with respect to noise is of fundamental importance. We thus 


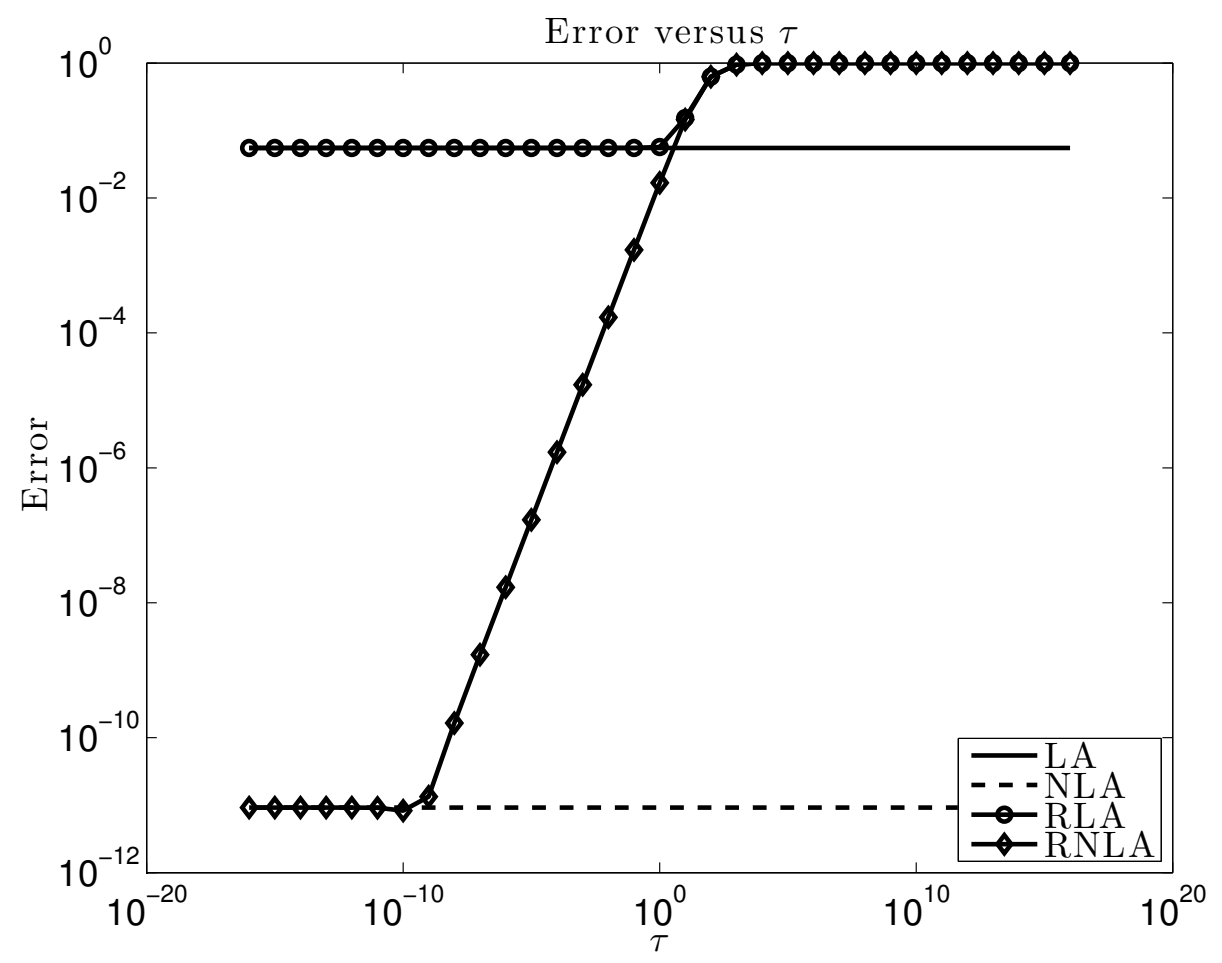

Figure 4: Plot of relative $L^{\infty}$ error in reconstructed solution (compared to the exact solution) versus the regularization parameter $\tau$ for the LA, NLA, RLA, and RNLA algorithms. Physical parameters: $\alpha=0.0, \beta=5.5, d=2 \pi, \bar{g}=-1.5$; profile parameters: $\varepsilon=0.01$, $R=10, \rho=10^{-16}$; numerical parameters: $N_{x}=32, N_{i}=8$. 


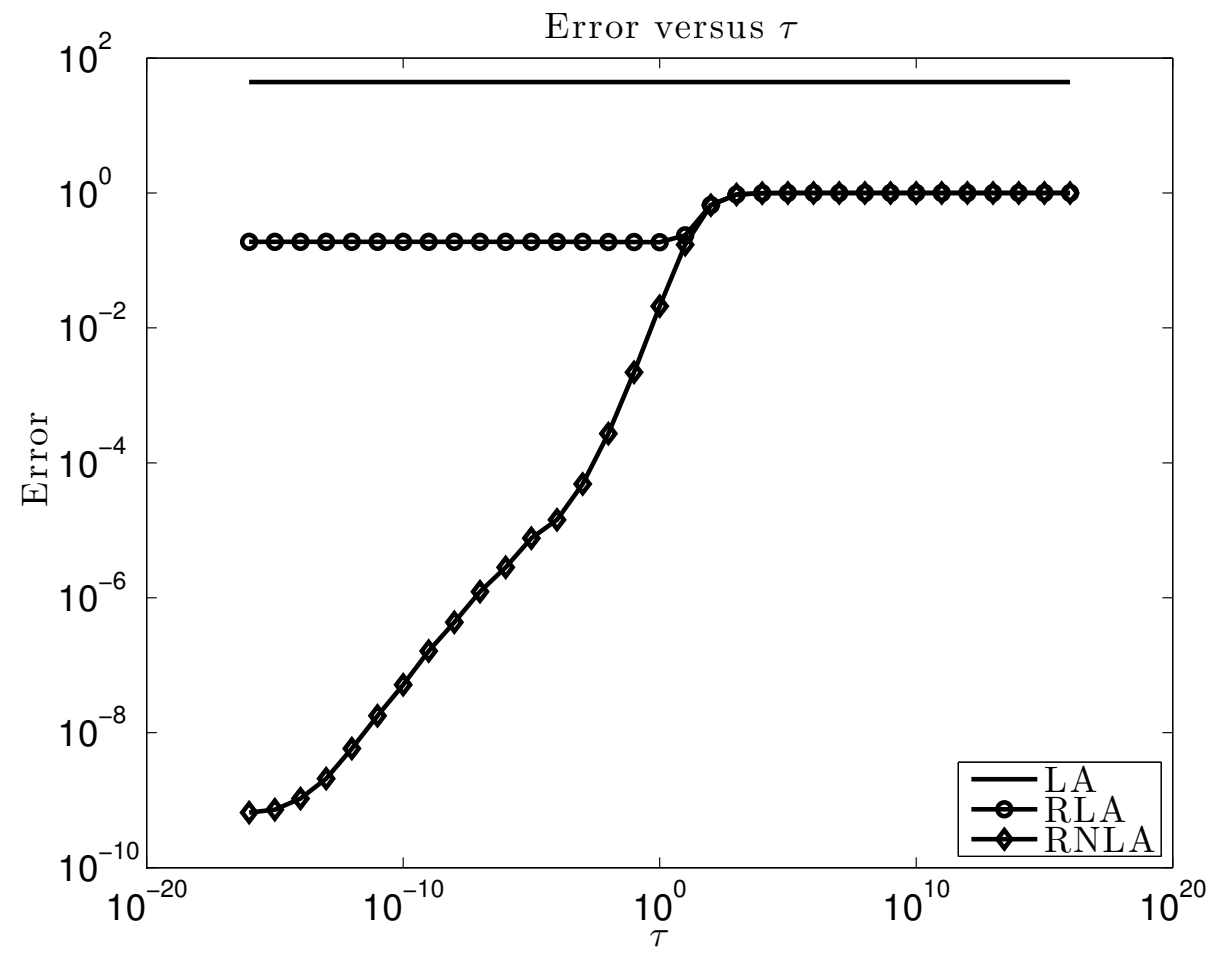

Figure 5: Plot of relative $L^{\infty}$ error in reconstructed solution (compared to the exact solution) versus the regularization parameter $\tau$ for the LA, RLA, and RNLA algorithms. Physical parameters: $\alpha=0.0, \beta=5.5, d=2 \pi, \bar{g}=-1.5$; profile parameters: $\varepsilon=0.03, R=20$, $\rho=10^{-16}$; numerical parameters: $N_{x}=128, N_{i}=8$. 


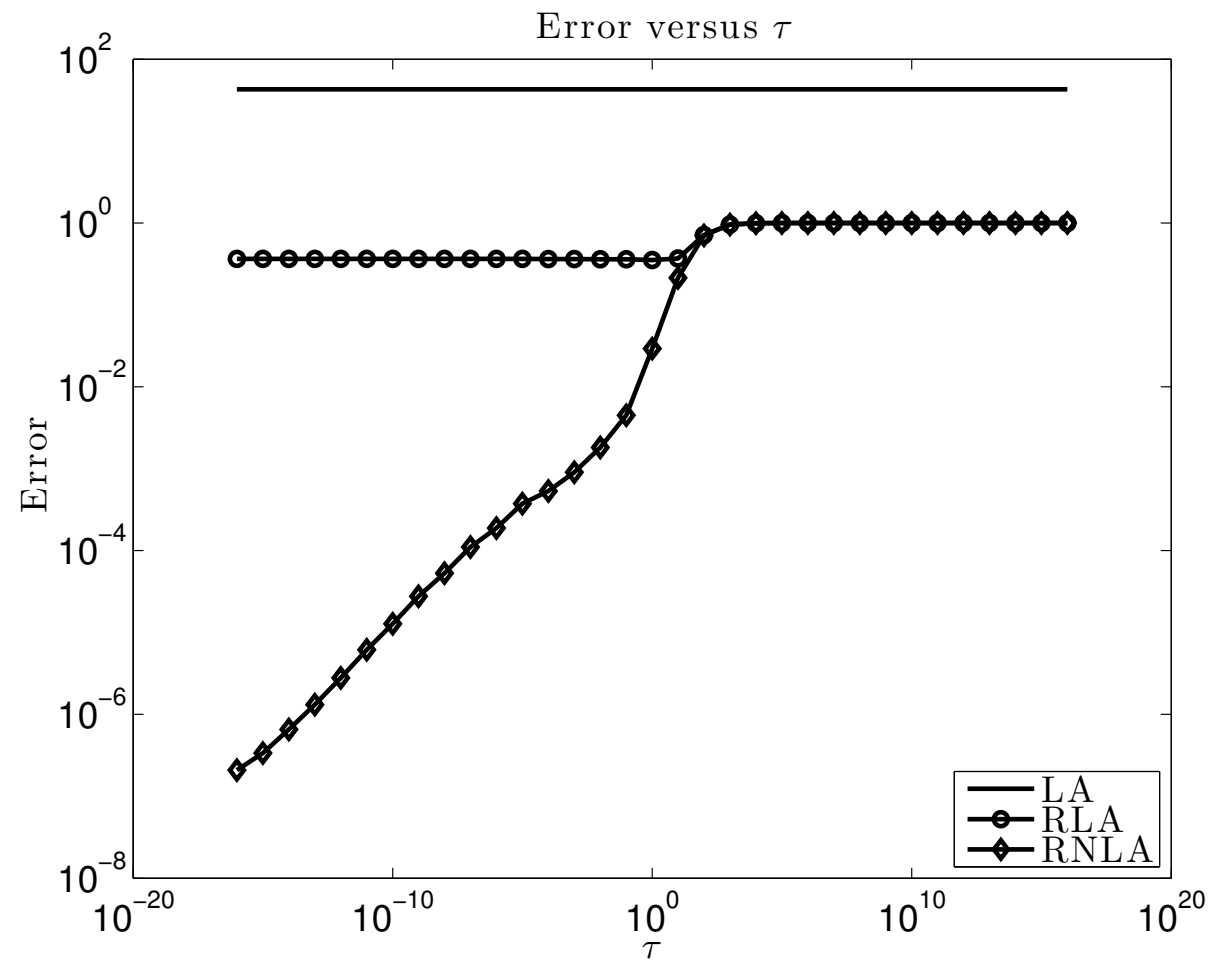

Figure 6: Plot of relative $L^{\infty}$ error in reconstructed solution (compared to the exact solution) versus the regularization parameter $\tau$ for the LA, RLA, and RNLA algorithms. Physical parameters: $\alpha=0.0, \beta=5.5, d=2 \pi, \bar{g}=-1.5$; profile parameters: $\varepsilon=0.05, R=30$, $\rho=10^{-16}$; numerical parameters: $N_{x}=128, N_{i}=8$. 
test the response of the algorithms to two types of random noise. Of course in real data such noise is present in all aspects of a reconstruction procedure from the specification of the incident radiation, to the assumptions that the layers are homogeneous (or even have a layered structure), to the measurements of the far-field pattern. To test our algorithms we take the point of view that the noise present in the incident wavenumber $(\alpha, \beta)$ is negligible, while we adhere to the model of a single acoustic layer with velocity constant up to a very small error. We introduce significant noise, however, to the far-field data we measure: $\left\{\hat{\eta}_{p}\right\}$.

For this we propose two approaches: "Absolute uniform noise"

$$
\hat{\chi}_{p}:=\hat{\eta}_{p}+a(2 \mathcal{X}-1), \quad \mathcal{X} \sim U(0,1),
$$

where $U(0,1)$ is a uniformly distributed random variable on the interval $(0,1)$, or "relative uniform noise"

$$
\hat{\theta}_{p}:=\{1+a(2 \mathcal{X}-1)\} \hat{\eta}_{p}, \quad \mathcal{X} \sim U(0,1) .
$$

For these simulations we use the rand command from MATLAB [MAT10] to generate the $U(0,1)$ samples (seeded, in every case, with the integer 100 so as to be reproducible). To simplify the exposition of our results we restrict ourselves to the profile (3.8) with $(\varepsilon=0.05, R=30)$ and noise amplitudes $a=10^{-3}, 10^{-2}, 10^{-1}$.

We now present results of simulations using this noise in Figure 7 for absolute noise, (6.1), and Figure 8 for relative noise, (6.2). Once again we notice the remarkable accuracy that our new RNLA algorithm can deliver, though, of course, the noise necessarily diminishes its performance greatly. In the absolute noise case we can realize relative accuracies of $10^{-3}$, $2 \times 10^{-3}$, and $9 \times 10^{-3}$ for values of $a=10^{-3}, 10^{-2}, 10^{-1}$, respectively (for $\tau=0.01$ in every case).

In the relative noise case we can realize relative accuracies of $5 \times 10^{-4}, 4 \times 10^{-3}$, and $4 \times 10^{-2}$ for values of $a=10^{-3}, 10^{-2}, 10^{-1}$, respectively. In these cases these are achieved for much smaller values of $\tau$ equal to $10^{-5}$ or $10^{-6}$.

\subsection{Moho Depth Variation Recovery}

In an effort to display the utility of our approach when applied to "real world" data we sought out a sample profile inspired by the Mohorovičić (Moho) discontinuity which separates the Earth's crust from the mantle underneath [ZK00]. For this we consulted the work of Zhu and Kanamori [ZK00] on measuring the depth of this discontinuity in southern California.

We took the estimated Moho depths given in [ZK00] beneath stations located between $-116^{\circ}$ and $-117^{\circ}$ longitude. We assumed that these points lie roughly along a line, and then smoothed and interpolated the resulting profile to obtain an equally sampled profile appropriate for this model (see Figure 11). The smoothing primarily compensates for the unequal sampling and deviations of the sample locations from a line.

Selecting the physical parameters (3.6):

$$
\alpha=0, \quad \beta=5.5, \quad d=2 \pi, \quad \bar{g}=-1.5,
$$

and the "Moho profile," we once again generate far-field data with our algorithm (3.2) with $N_{x}=128$ and $N=12$. (We point out that these parameter choices represent illumination from a single direction from above, as opposed to the more realistic situation of multiple directions from below.) These data were relatively polluted with uniformly distributed noise of amplitude $a=10^{-1},(6.2)$, and given to our RLA and RNLA algorithms. The results 


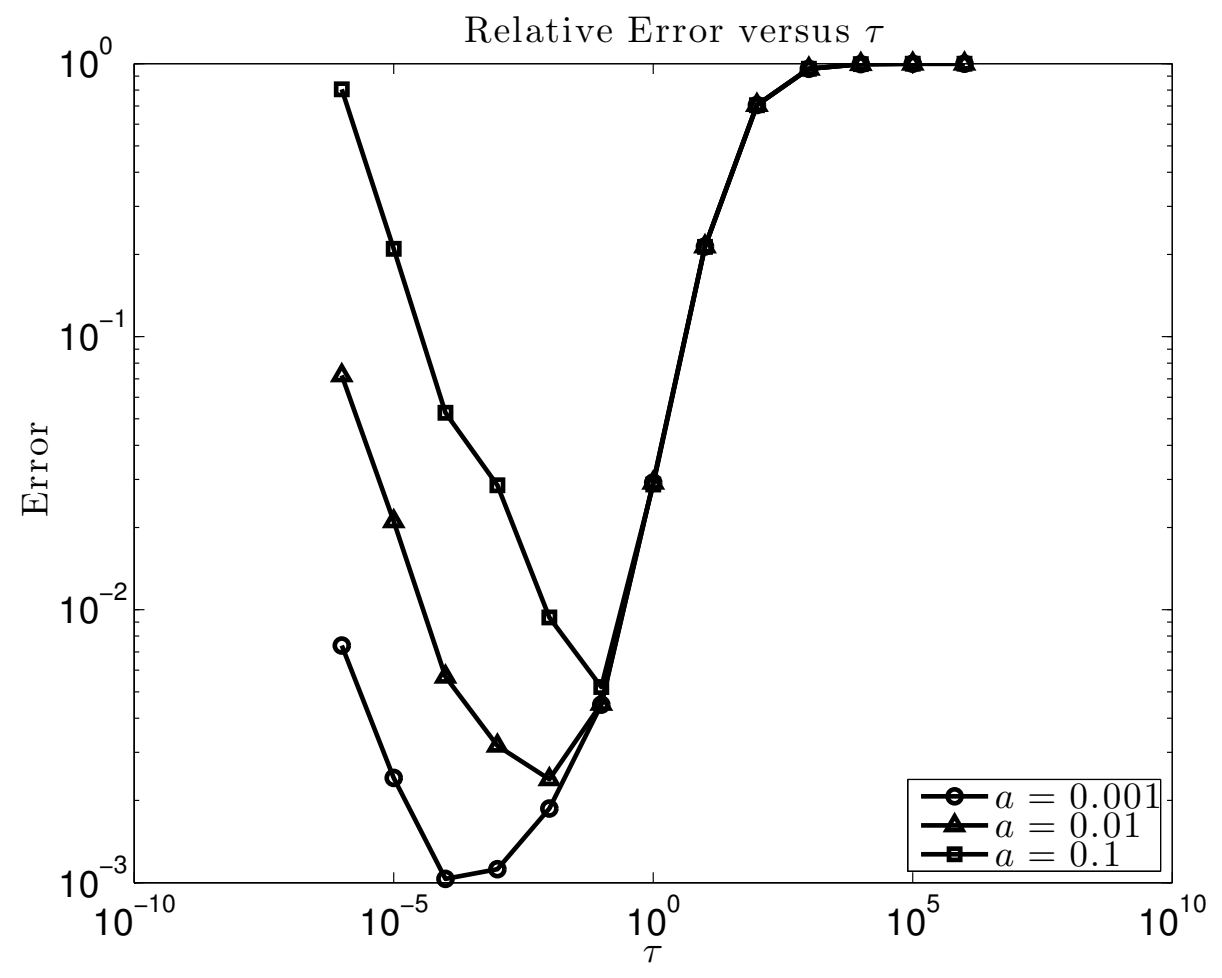

Figure 7: Relative $L^{\infty}$ error, versus regularization parameter $\tau$, in RNLA approximation of the true interface shape with far-field data, $\hat{\eta}_{p}$, polluted by absolute, uniformly distributed noise, (6.1), of amplitudes $a=10^{-3}, 10^{-2}, 10^{-1}$. Physical parameters: $\alpha=0.0, \beta=5.5$, $d=2 \pi, \bar{g}=-1.5$; profile parameters: $\varepsilon=0.05, R=30, \rho=10^{-16}$; numerical parameters: $N_{i}=8, N_{x}=128$. 


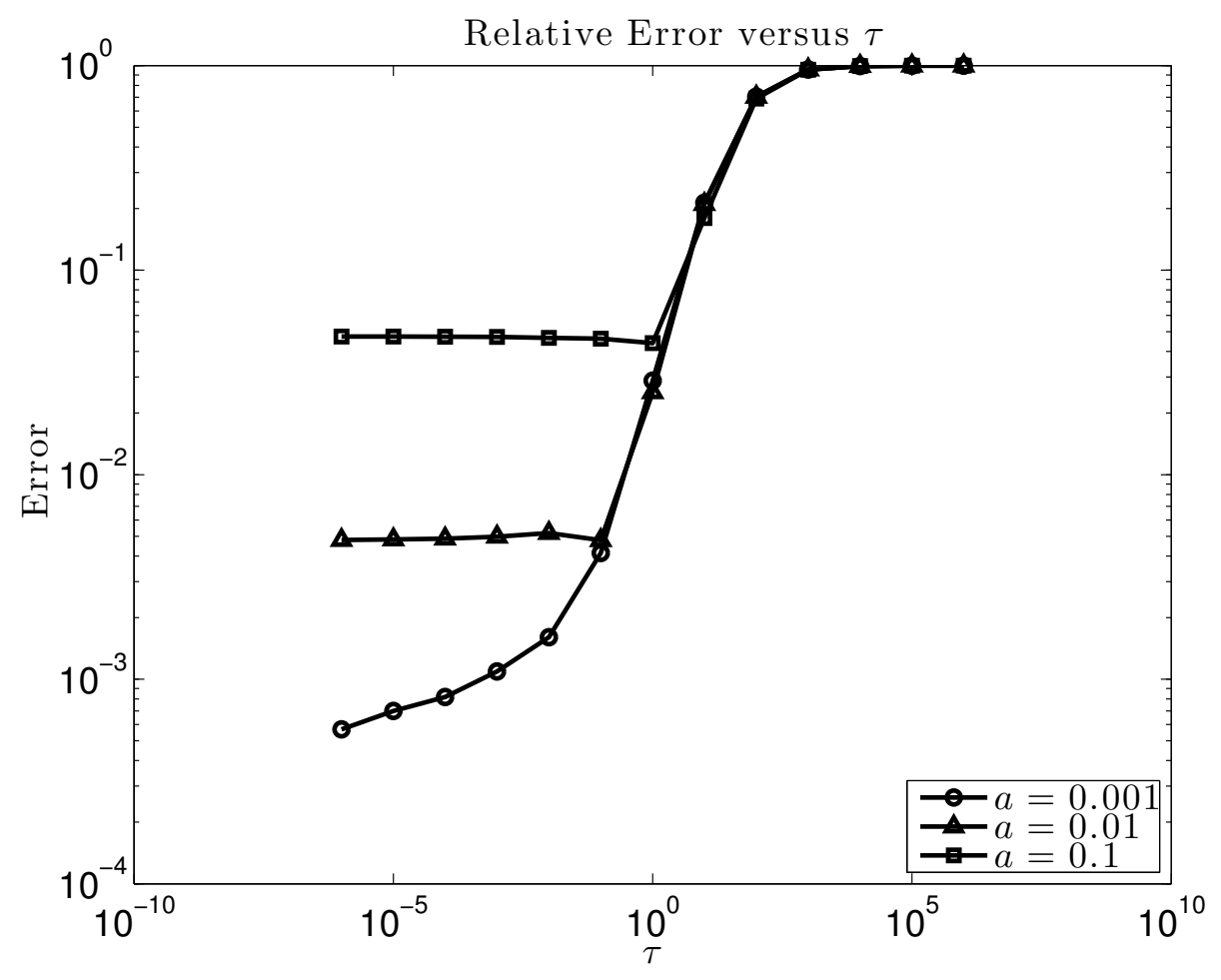

Figure 8: Relative $L^{\infty}$ error, versus regularization parameter $\tau$, in RNLA approximation of the true interface shape with far-field data, $\hat{\eta}_{p}$, polluted by relative, uniformly distributed noise, (6.2), of amplitudes $a=10^{-3}, 10^{-2}, 10^{-1}$. Physical parameters: $\alpha=0.0, \beta=5.5$, $d=2 \pi, \bar{g}=-1.5$; profile parameters: $\varepsilon=0.05, R=30, \rho=10^{-16}$; numerical parameters: $N_{i}=8, N_{x}=128$. 


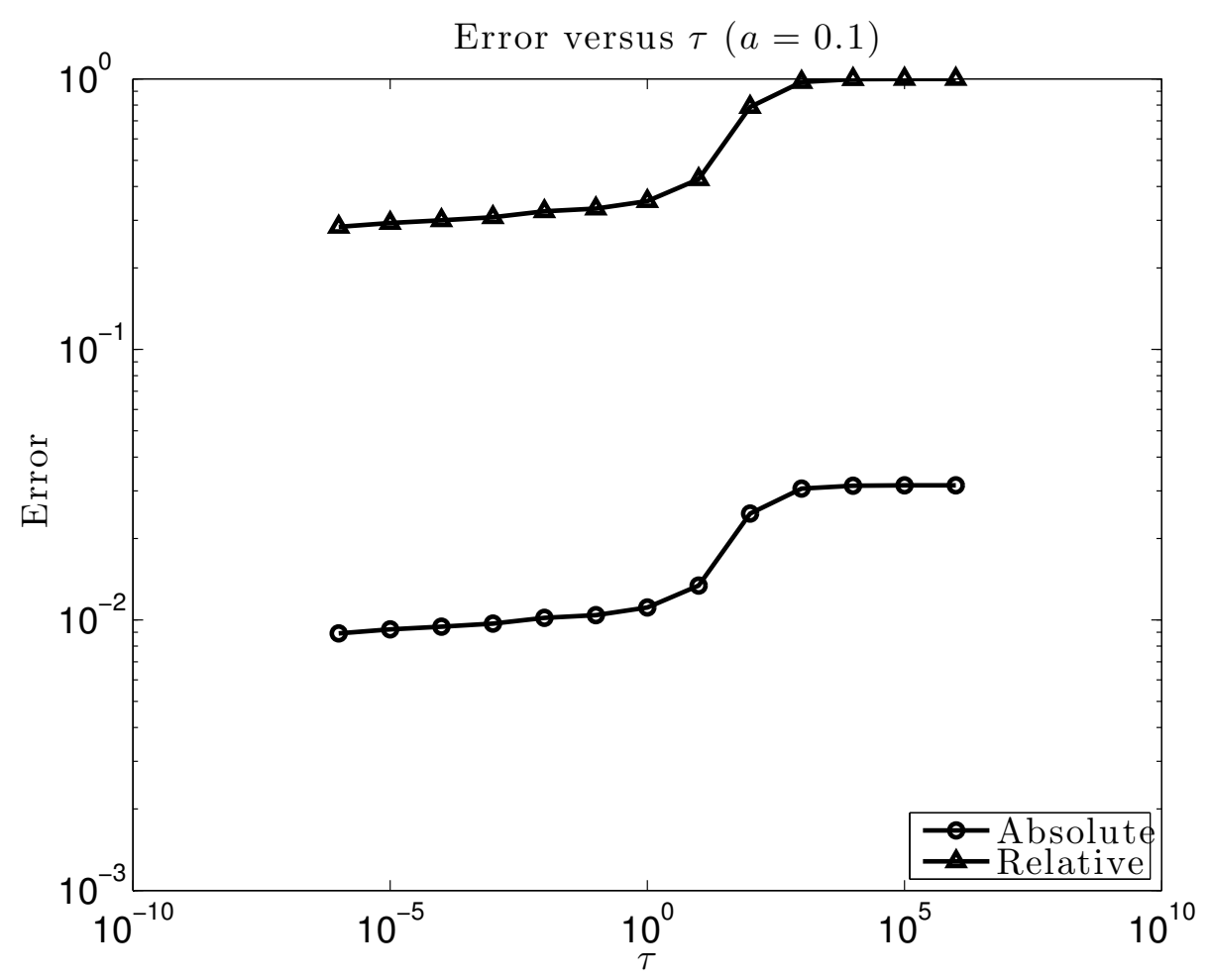

Figure 9: Absolute and relative $L^{\infty}$ error, versus regularization parameter $\tau$, in RNLA approximation of the true Moho interface shape with far-field data, $\hat{\eta}_{p}$, polluted by relative, uniformly distributed noise, (6.2), of amplitude $a=10^{-1}$. Physical parameters: $\alpha=0.0$, $\beta=5.5, d=2 \pi, \bar{g}=-1.5$; numerical parameters: $N_{i}=8, N_{x}=128$.

are tabulated in Figure 9 for the RNLA method, and the actual reconstructions (in the case $\tau=10^{-6}$ ) together with the exact profile are reproduced in Figure 11. Despite the aperiodicity of the Moho profile, we see the impressive capabilities of our new approach in recovering the Moho discontinuity. As we measure in $L^{\infty}$, almost all of the error is due to the non-periodicity in this more realistic simulation. We make this more precise in Figure 10 with $L^{2}$ measurements.

\section{Acknowledgments}

AM gratefully acknowledges support from the National Science Foundation through grant No. DMS-1115406, and the sponsors of the Earth Resources Laboratory.

DPN gratefully acknowledges support from the National Science Foundation through grant No. DMS-1115333, and the Department of Energy under Award No. DE-SC0001549.

Disclaimer: This report was prepared as an account of work sponsored by an agency of the United States Government. Neither the United States Government nor any agency thereof, nor any of their employees, make any warranty, express or implied, or assumes any legal liability or responsibility for the accuracy, completeness, or usefulness of any information, apparatus, product, or process disclosed, or represents that its use would not infringe privately owned rights. Reference herein to any specific commercial product, process, or service by trade name, trademark, manufacturer, or otherwise does not necessarily constitute 


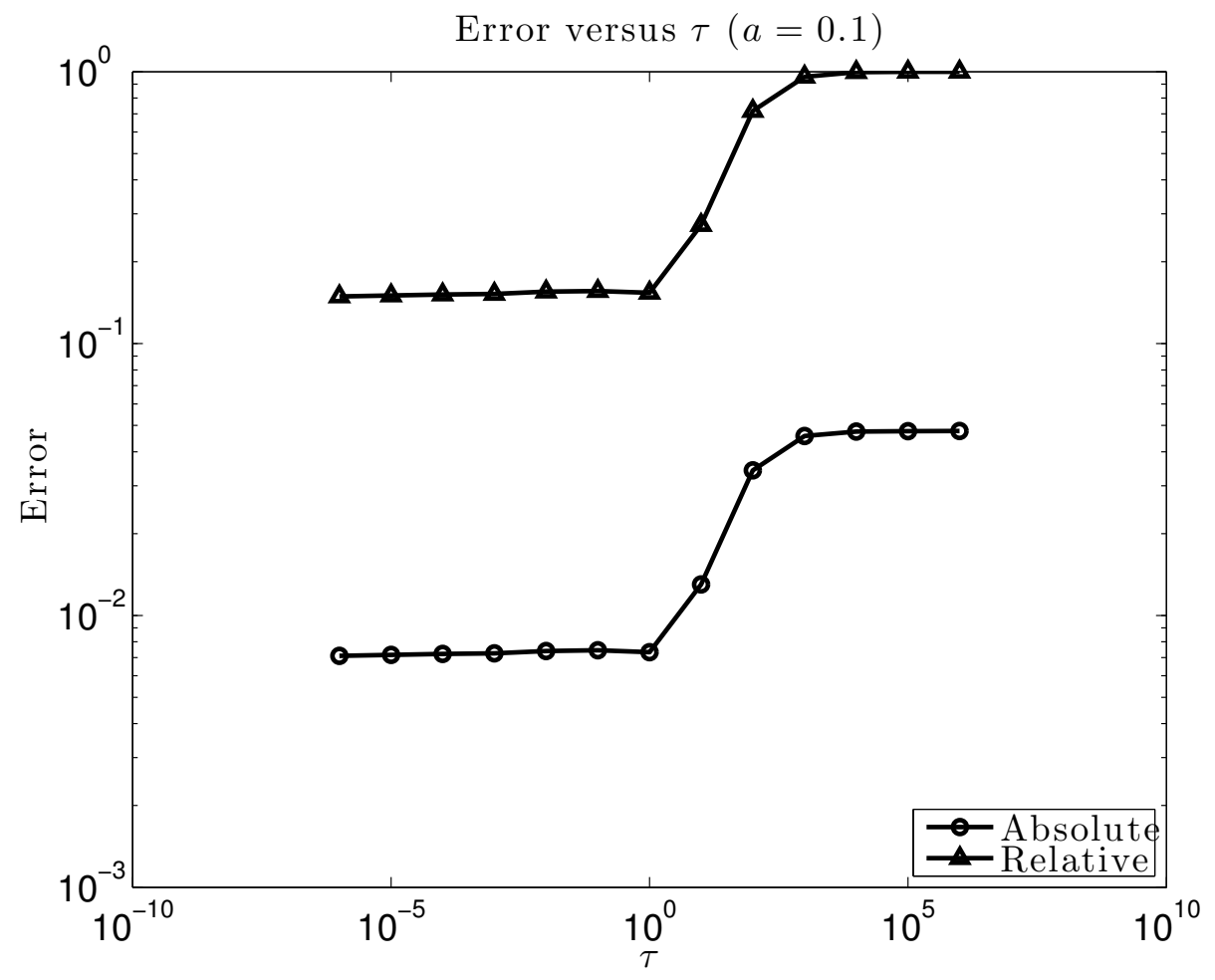

Figure 10: Absolute and relative $L^{2}$ error, versus regularization parameter $\tau$, in RNLA approximation of the true Moho interface shape with far-field data, $\hat{\eta}_{p}$, polluted by relative, uniformly distributed noise, (6.2), of amplitude $a=10^{-1}$. Physical parameters: $\alpha=0.0$, $\beta=5.5, d=2 \pi, \bar{g}=-1.5$; numerical parameters: $N_{i}=8, N_{x}=128$. 


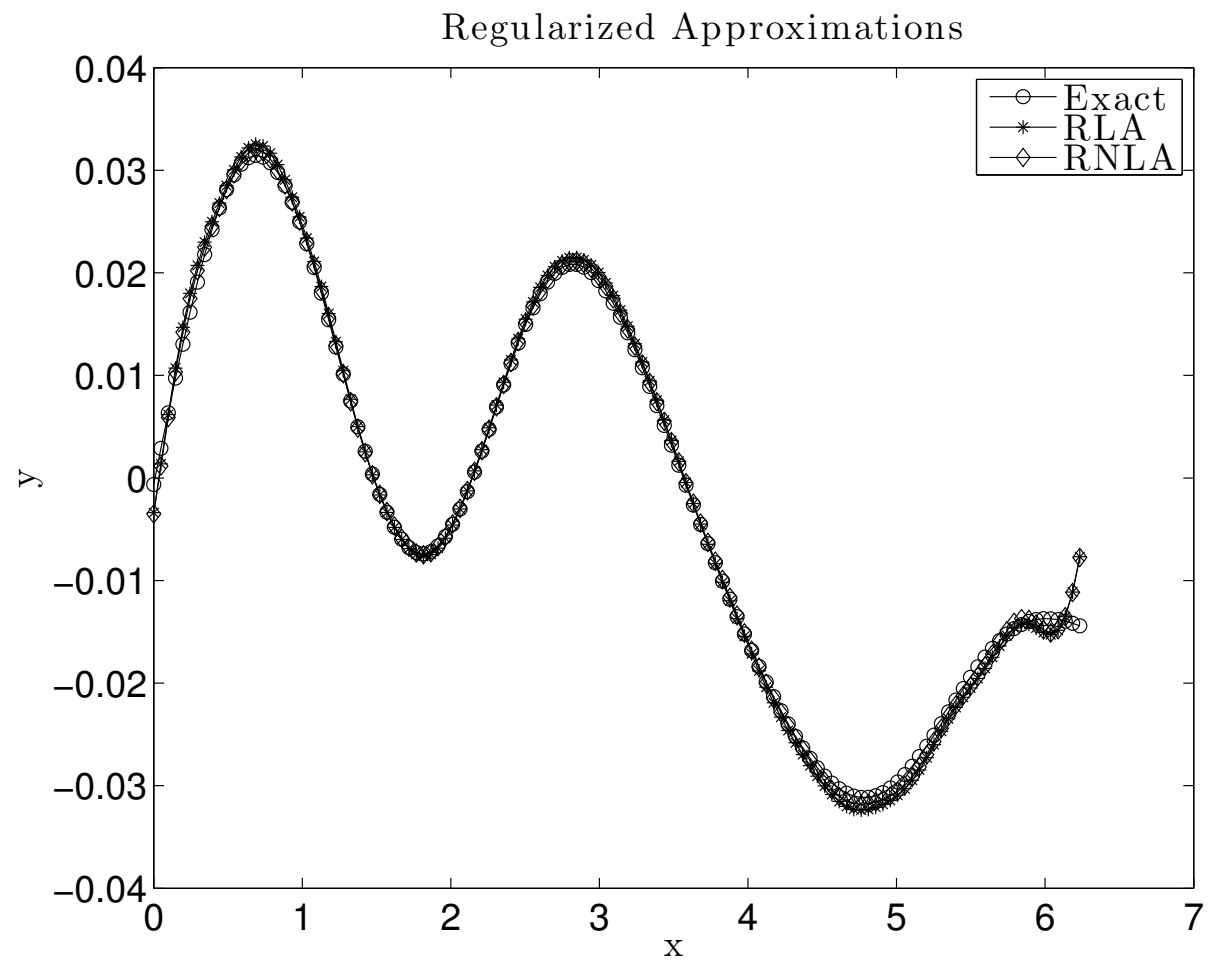

Figure 11: Plot of experimentally inspired Moho layer shape together with RLA and RNLA reconstructions in the presence of relative, uniformly distributed noise, (6.2), of amplitude $a=10^{-1}$. Physical parameters: $\alpha=0.0, \beta=5.5, d=2 \pi, \bar{g}=-1.5$; numerical parameters: $N_{i}=8, N_{x}=128$. 
or imply its endorsement, recommendation, or favoring by the United States Government or any agency thereof. The views and opinions of authors expressed herein do not necessarily state or reflect those of the United States Government or any agency thereof.

\section{A The Sound-Hard Case}

To complement our developments in $\S 2$ we note that an acoustic wave interacting with a two-dimensional "sound hard" periodic grating has scattered field described by the Neumann problem

$$
\begin{array}{ll}
\Delta u+k^{2} u=0 & y>\bar{g}+g(x) \\
\partial_{y} u-\left(i \beta_{D}\right) u=0 & y=0 \\
\left(\partial_{\tilde{N}} u\right)(x, \bar{g}+g(x))=\psi(x) & \\
u(x+d, y)=e^{i \alpha d} u(x, y), &
\end{array}
$$

where

$$
\tilde{N}:=\left(-\partial_{x} g, 1\right)^{T}
$$

and

$$
\psi(x):=-\left(i \beta+i \alpha\left(\partial_{x} g\right)\right) e^{i \alpha x-i \beta(\bar{g}+g(x))} .
$$

For a surface formulation we define the exterior Neumann trace

$$
U^{\prime}(x):=-\left(\partial_{\tilde{N}} u\right)(x, \bar{g}+g(x)),
$$

so that (A.1) becomes

$$
-U^{\prime}=\psi
$$

Introducing the "Neumann Propagation Operator" (NPO) $T$

$$
T(\bar{g}+g)\left[U^{\prime}(x)\right]:=\tilde{u}(x),
$$

c.f. (3.1), the forward problem becomes a solution of the equation

$$
\tilde{u}(x)=T\left[U^{\prime}\right]=-T[\psi(x)]=-T(g)[\psi(x ; g)] .
$$

Again, setting $g(x)=\varepsilon f(x)$, expanding

$$
T=T(\varepsilon f)=\sum_{n=0}^{\infty} T_{n}(f) \varepsilon^{n}, \quad \psi=\psi(x ; \varepsilon f)=\sum_{n=0}^{\infty} \psi_{n}(x) \varepsilon^{n},
$$

and

$$
\tilde{u}=\tilde{u}(x ; \varepsilon)=\sum_{n=0}^{\infty} \tilde{u}_{n}(x) \varepsilon^{n},
$$

we insert these into (A.3). The $n$-th correction $\tilde{u}_{n}$ solves

$$
\tilde{u}_{n}=-\sum_{m=0}^{n} T_{n-m}\left[\psi_{m}\right]
$$


and we again obtain a highly accurate approximation

$$
\tilde{u}^{N}(x ; \varepsilon):=\sum_{n=0}^{N} \tilde{u}_{n}(x) \varepsilon^{n},
$$

which may be summed directly (Taylor summation) or via numerical analytic continuation methods (Padé summation).

Finally, to enable our perturbation strategies for both the forward and inverse problems, we note the following expansions which can be derived as $\zeta_{n}$ and $P_{n}$ were in $\S 3.1$. At order zero

$$
\begin{aligned}
\psi_{0}(x) & =-i \beta e^{-i \beta \bar{g}} e^{i \alpha x} \\
T_{0} & =-\frac{1}{\left(i \beta_{D}\right)} e^{-i \beta_{D} \bar{g}},
\end{aligned}
$$

and at order $n>0$

$$
\begin{aligned}
\psi_{n}(x) & =\left\{-i \beta(-i \beta)^{n} F_{n}(x)-i \alpha\left(\partial_{x} f\right)(-i \beta)^{n-1} F_{n-1}(x)\right\} e^{i \alpha x} e^{-i \beta \bar{g}} \\
T_{n} & =\sum_{m=0}^{n-1} T_{m}(f)\left[\left\{k^{2} F_{n-m}+\partial_{x} F_{n-m} \partial_{x}\right\}\left(i \beta_{D}\right)^{n-m-2}\right] .
\end{aligned}
$$

\section{References}

[AG05] T. Arens and N. Grinberg. A complete factorization method for scattering by periodic surfaces. Computing, 75(2-3):111-132, 2005.

[AKY06] I. Akduman, R. Kress, and A. Yapar. Iterative reconstruction of dielectric rough surface profiles at fixed frequency. Inverse Problems, 22(3):939-954, 2006.

[BE03] G. Bruckner and J. Elschner. A two-step algorithm for the reconstruction of perfectly reflecting periodic profiles. Inverse Problems, 19(2):315-329, 2003.

[BE05] G. Bruckner and J. Elschner. The numerical solution of an inverse periodic transmission problem. Math. Methods Appl. Sci., 28(7):757-778, 2005.

[BGM96] G. Baker, Jr. and P. Graves-Morris. Padé approximants. Cambridge University Press, Cambridge, second edition, 1996.

[BL82] L. Brekhovskikh and Y. Lysanov. Fundamentals of Ocean Acoustics. SpringerVerlag, Berlin, 1982.

[BR93a] O. Bruno and F. Reitich. Numerical solution of diffraction problems: A method of variation of boundaries. J. Opt. Soc. Am. A, 10(6):1168-1175, 1993.

[BR93b] O. Bruno and F. Reitich. Numerical solution of diffraction problems: A method of variation of boundaries. II. Finitely conducting gratings, Padé approximants, and singularities. J. Opt. Soc. Am. A, 10(11):2307-2316, 1993.

[BR93c] O. Bruno and F. Reitich. Numerical solution of diffraction problems: A method of variation of boundaries. III. Doubly periodic gratings. J. Opt. Soc. Am. A, 10(12):2551-2562, 1993. 
[BR01] O. Bruno and F. Reitich. High-order boundary perturbation methods. In Mathematical Modeling in Optical Science, volume 22, pages 71-109. SIAM, Philadelphia, PA, 2001. Frontiers in Applied Mathematics Series.

[CHQZ88] C. Canuto, Y. Hussaini, A. Quarteroni, and T. Zang. Spectral methods in fluid dynamics. Springer-Verlag, New York, 1988.

[CK83] D. Colton and R. Kress. Integral equation methods in scattering theory. Pure and Applied Mathematics (New York). John Wiley \& Sons Inc., New York, 1983. A Wiley-Interscience Publication.

[CK98] D. Colton and R. Kress. Inverse acoustic and electromagnetic scattering theory. Springer-Verlag, Berlin, second edition, 1998.

[CS93] W. Craig and C. Sulem. Numerical simulation of gravity waves. Journal of Computational Physics, 108:73-83, 1993.

[EHR04] J. Elschner, G. Hsiao, and A. Rathsfeld. Grating profile reconstruction based on finite elements and optimization techniques. SIAM J. Appl. Math., 64(2):525$545,2003 / 04$.

[Het02] F. Hettlich. Iterative regularization schemes in inverse scattering by periodic structures. Inv. Prob., 18:701-714, 2002.

[HN05] B. Hu and D. Nicholls. Analyticity of Dirichlet-Neumann operators on Hölder and Lipschitz domains. SIAM J. Math. Anal., 37(1):302-320, 2005.

[HN10] B. Hu and D. Nicholls. The domain of analyticity of Dirichlet-Neumann operators. Proceedings of the Royal Society of Edinburgh A, 140(2):367-389, 2010.

[IR99] K. Ito and F. Reitich. A high-order perturbation approach to profile reconstruction. I. Perfectly conducting gratings. Inverse Problems, 15(4):1067-1085, 1999 .

[KR09] H. Kurkcu and F. Reitich. Stable and efficient evaluation of periodized Green's functions for the Helmholtz equation at high frequencies. J. Comput. Phys., 228(1):75-95, 2009.

[Kre99] R. Kress. Linear integral equations. Springer-Verlag, New York, second edition, 1999.

[KT94] P. Kaczkowski and E. Thorsos. Application of the operator expansion method to scattering from one-dimensional moderately rough Dirichlet random surfaces. J. Acoust. Soc. Am., 96(2):957-972, 1994.

[KT00] R. Kress and T. Tran. Inverse scattering for a locally perturbed half-plane. Inverse Problems, 16(5):1541-1559, 2000.

[LG11] C. Lahcene and P. Gaitan. Reconstruction of the interface between two-layered media using far field measurements. Inverse Problems, 27(7), 2011.

[MAT10] MATLAB. version 7.10 .0 (R2010a). The MathWorks Inc., Natick, Massachusetts, 2010. 
[Mil91a] M. Milder. An improved formalism for rough-surface scattering of acoustic and electromagnetic waves. In Proceedings of SPIE - The International Society for Optical Engineering (San Diego, 1991), volume 1558, pages 213-221. Int. Soc. for Optical Engineering, Bellingham, WA, 1991.

[Mil91b] M. Milder. An improved formalism for wave scattering from rough surfaces. $J$. Acoust. Soc. Am., 89(2):529-541, 1991.

[Mil96a] M. Milder. An improved formalism for electromagnetic scattering from a perfectly conducting rough surface. Radio Science, 31(6):1369-1376, 1996.

[Mil96b] M. Milder. Role of the admittance operator in rough-surface scattering. $J$. Acoust. Soc. Am., 100(2):759-768, 1996.

[MN11a] A. Malcolm and D. Nicholls. A boundary perturbation method for recovering interface shapes in layered media. Inverse Problems, 27(9):095009, 2011.

[MN11b] A. Malcolm and D. Nicholls. A field expansions method for scattering by periodic multilayered media. Journal of the Acoustical Society of America, 129(4):17831793, 2011.

[MS91] M. Milder and T. Sharp. Efficient computation of rough surface scattering. In Mathematical and numerical aspects of wave propagation phenomena (Strasbourg, 1991), pages 314-322. SIAM, Philadelphia, PA, 1991.

[MS92] D. Milder and T. Sharp. An improved formalism for rough surface scattering. ii: Numerical trials in three dimensions. J. Acoust. Soc. Am., 91(5):2620-2626, 1992.

[NR01a] D. Nicholls and F. Reitich. A new approach to analyticity of Dirichlet-Neumann operators. Proc. Roy. Soc. Edinburgh Sect. A, 131(6):1411-1433, 2001.

[NR01b] D. Nicholls and F. Reitich. Stability of high-order perturbative methods for the computation of Dirichlet-Neumann operators. J. Comput. Phys., 170(1):276$298,2001$.

[NR03] D. Nicholls and F. Reitich. Analytic continuation of Dirichlet-Neumann operators. Numer. Math., 94(1):107-146, 2003.

[NR04a] D. Nicholls and F. Reitich. Shape deformations in rough surface scattering: Cancellations, conditioning, and convergence. J. Opt. Soc. Am. A, 21(4):590605, 2004.

[NR04b] D. Nicholls and F. Reitich. Shape deformations in rough surface scattering: Improved algorithms. J. Opt. Soc. Am. A, 21(4):606-621, 2004.

[NR08] D. Nicholls and F. Reitich. Boundary perturbation methods for highfrequency acoustic scattering: Shallow periodic gratings. J. Acoust. Soc. Amer., 123(5):2531-2541, 2008.

[NT08] D. Nicholls and M. Taber. Joint analyticity and analytic continuation for Dirichlet-Neumann operators on doubly perturbed domains. J. Math. Fluid Mech., 10(2):238-271, 2008. 
[NT09] D. Nicholls and M. Taber. Detection of ocean bathymetry from surface wave measurements. Euro. J. Mech. B/Fluids, 28(2):224-233, 2009.

[NW01] F. Natterer and F. Wübbeling. Mathematical methods in image reconstruction. SIAM Monographs on Mathematical Modeling and Computation. Society for Industrial and Applied Mathematics (SIAM), Philadelphia, PA, 2001.

[NW06] J. Nocedal and S. Wright. Numerical optimization. Springer Series in Operations Research and Financial Engineering. Springer, New York, second edition, 2006.

[Pet80] R. Petit, editor. Electromagnetic theory of gratings. Springer-Verlag, Berlin, 1980 .

[Shu02] P. Shull. Nondestructive Evaluation: Theory, Techniques, and Applications. Marcel Dekker, 2002.

[TKS85] L. Tsang, J. A. Kong, and R. T. Shin. Theory of Microwave Remote Sensing. Wiley, New York, 1985.

[Vor99] A. Voronovich. Wave scattering from rough surfaces. Springer-Verlag, Berlin, second edition, 1999.

[ZK00] L. Zhu and H. Kanamori. Moho depth variation in southern California from teleseismic receiver functions. J. Geophys. Res., 105(B2):2969-2980, 2000. 